\title{
Amplified spontaneous emission in the extreme ultraviolet by expanding xenon clusters
}

\author{
Andrei Benediktovitch $\odot,{ }^{1, *}$ Laurent Mercadier, ${ }^{2}$ Olivier Peyrusse, ${ }^{3}$ Andreas Przystawik, ${ }^{1}$ Tim Laarmann ${ }^{\circ},{ }^{1,4}$ \\ Bruno Langbehn $\odot,{ }^{5}$ Cédric Bomme, ${ }^{1}$ Benjamin Erk $\odot,{ }^{1}$ Jonathan Correa, ${ }^{1}$ Caroline Mossé, ${ }^{3}$ Daniel Rolles $\odot,{ }^{1,6}$ \\ Sven Toleikis, ${ }^{1}$ Maximilian Bucher $\odot,{ }^{7}$ Christoph F. O. Bostedt, ${ }^{8}$ Alvaro Sanchez-Gonzalez $\odot,{ }^{9}$ Stepan Dobrodey, ${ }^{10}$ \\ Michael A. Blessenohl $\odot,{ }^{10}$ Alexander Nelde $\odot{ }^{5}{ }^{2}$ Maria Müller, ${ }^{5}$ Daniela Rupp ${ }^{\circ},{ }^{5,11}$ Thomas Möller, ${ }^{5}$ \\ José R. Crespo López-Urrutia, ${ }^{10}$ and Nina Rohringer ${ }^{1,12}$ \\ ${ }^{1}$ Deutsches Elektronen-Synchrotron DESY, Hamburg 22607, Germany \\ ${ }^{2}$ European XFEL GmbH, Schenefeld 22869, Germany \\ ${ }^{3}$ Aix-Marseille Université, CNRS UMR 7345, PIIM, Marseille, France \\ ${ }^{4}$ Centre for Ultrafast Imaging CUI, Hamburg 22761, Germany \\ ${ }^{5}$ Institut für Optik und Atomare Physik, Technische Universität Berlin, Berlin 10623, Germany \\ ${ }^{6}$ J. R. Macdonald Laboratory, Department of Physics, Kansas State University, Manhattan, Kansas 66506-2601, USA \\ ${ }^{7}$ Linac Coherent Light Source, SLAC National Accelerator Laboratory, Menlo Park, California 94025, USA \\ ${ }^{8}$ Argonne National Laboratory, Lemont, Illinois 60439, USA \\ ${ }^{9}$ Imperial College, Department of Physics, London SW7 2AZ, United Kingdom \\ ${ }^{10}$ Max-Plank-Institut für Kernphysik, Heidelberg, 69117 Heidelberg, Germany \\ ${ }^{11}$ Department of Physics, ETH Zurich, 8093 Zurich, Switzerland \\ ${ }^{12}$ Department of Physics, Universität Hamburg, Hamburg 22761, Germany
}

(Received 27 January 2020; revised manuscript received 27 April 2020; accepted 26 May 2020; published 22 June 2020)

\begin{abstract}
Focused short-wavelength free-electron laser (FEL) pulses interacting with gas phase samples can induce by inner-shell ionization a short-lived population inversion, followed by coherent collective emission of directed, short, and strong radiation bursts. We extend our studies into the warm-dense matter (WDM) regime by investigating the nanoplasmas produced in an ensemble of nanometer-sized clusters by FEL irradiation. Here, additional pathways can also lead to strong, laserlike emission: Electron-ion collisions can yield a long-lived population inversion, and subsequent amplified spontaneous emission. We observe amplified spontaneous emission (ASE) in the extreme ultraviolet in xenon clusters excited by soft x-ray FEL pulses, we diagnose the generated nanoplasmas by fluorescence spectroscopy, and we study under various cluster and FEL parameters the directed ASE from the $\mathrm{Xe}^{2+} 65 \mathrm{~nm}$ line. We show its exponential increase as a function of FEL irradiation power, and an accompanying collisional broadening of the emission spectra. These findings are corroborated by extensive numerical simulations based on theory, combining detailed hydrodynamic and kinetic simulations with time-dependent calculations of radiation transport, amplification, and collective emission in the WDM nanoplasma. Our theoretical findings underline that population inversion is due to electron-ion collisions and that the observed decoherence processes can be empirically characterized by a phenomenological decoherence time in the range of 100-200 fs.
\end{abstract}

DOI: 10.1103/PhysRevA.101.063412

\section{INTRODUCTION}

Short-wavelength free-electron lasers (FELs) enable the preparation and study of matter under extreme conditions [1-3]. By focusing the FEL beam onto a target, each atom within a sample can absorb one or several high-energy photons within the duration of the FEL pulse [4,5], thus leaving the system in a highly excited state, out of equilibrium [6-9]. In particular, the ultrafast plasma production by inner-shell photoionization can lead to sizable population inversion and collective stimulated emission. Stimulated x-ray emission and superfluorescence have been observed in FEL generated plasmas in the gas [10,11], liquid [12], and solid

\footnotetext{
*andrei.benediktovitch@ desy.de
}

phases [13], resulting in directed $\mathrm{x}$-ray bursts featuring exponential growth in emission yield as a function of pump power. Saturated x-ray emission, for which collective radiative decay outpaces electronic Auger relaxation, has been observed in several cases $[11,12]$. In the $\mathrm{x}$-ray region, this coherent emission follows the interaction with the FEL pulse [14,15] with only a few fs delay.

Transitions in the extreme ultraviolet (XUV) spectral range have typical radiative lifetimes in the range of ns. Those that involve inner-shell holes have to compete with fs to sub-fs Auger- or Coster-Kronig decays, respectively. This inhibits the buildup of macroscopic polarization and collective emission, and thus far stimulated XUV emission involving an inner-shell transition has not been observed. Superfluorescent XUV emission promptly following interaction with an FEL pulse has been recently observed in $\mathrm{Xe}$ gas [16] for $\mathrm{Xe}^{2+}$ 
transitions of 65 and $68 \mathrm{~nm}$. In that scheme, a long-lived population inversion between valence excited states was prepared by Auger decay, following inner-shell (4d) ionization of Xe. Here, we demonstrate that the interaction of an FEL pulse with Xe clusters can lead to a secondary time window of collective XUV emission approximately $1-3$ ps following the interaction with the FEL pulse. At this stage, the plasma is governed by electron-ion collisions in the evolving warm-dense matter (WDM) state [17-19], a transitional state between solid and plasma. Within this time, the population inversion is induced by electron collisions (predominantly electron-impact excitation and three-body recombination). Compared to the gas phase atomic experiments [16], we observe a more than tenfold increase of the population inversion in the cluster target, but a smaller emission yield because of strong collisional dephasing: At similar conditions (optical density, FEL parameters), the directed XUV emission lines at 65 and $68 \mathrm{~nm}$ of clusters are characterized by a considerably larger linewidth, and an emission yield several orders of magnitude smaller (but still several orders of magnitude larger than pure fluorescence) than in the gas phase. We attribute the cluster emission to collisionally pumped amplified spontaneous emission (ASE). A quantitative comparison of the experimental data to our theoretical approach allows us to assign a characteristic, phenomenological decoherence time associated with the electronion collisions.

Before the invention of (soft) x-ray FELs, hot-dense plasma or WDM were the key to producing coherent shortwavelength radiation. Warm/hot plasmas produced in capillary electrical discharges, or prepared in well-defined states by high-intensity optical lasers, were the basis for XUV and soft X-ray laserlike emission in table-top-like settings [20]. Collisional recombination or electron-impact excitation in WDM are the key processes to generate a population inversion in a long, extended target, ultimately resulting in laserlike emission. Unlike traditional table-top XUV and soft $\mathrm{x}$-ray laser sources, which typically involve hot dense plasma transitions of highly charged ions (Ne- or Ni-like ions), here we observe lasing in low charge states. Our work closely connects to optical-laser driven table-top soft x-ray lasers and plasma generated $x$-ray sources [21-23]. In this field, much focus lies on the target design - structured targets on the nanoscale, such as nanowire arrays [24,25], foams [26], nanoparticle targeted surfaces [27,28], and clusters [29-33] can exhibit enhanced emission features. The understanding of the plasma evolution of such sources is, however, crucial to the gain and emission characteristics of these sources. Our detailed study on amplified spontaneous emission and plasma evolution in a nanometer-sized cluster target, therefore, directly connects to this wider range of activities. In addition, the study of XUV emission of cluster jets is of the utmost importance for XUV source development [34] for 13.5-nm lithography, which is currently based on micrometer-sized tin droplets [35].

The paper is organized as follows: In Sec. II, we describe the experimental layout and discuss the experimental data in several scenarios. In Sec. III, our simulations of the cluster evolution, based on hydrodynamic-atomic code, are described, and mechanisms leading to population inversion are discussed. In Sec. IV, we derive the theory for amplified spontaneous emission under the relevant experimental

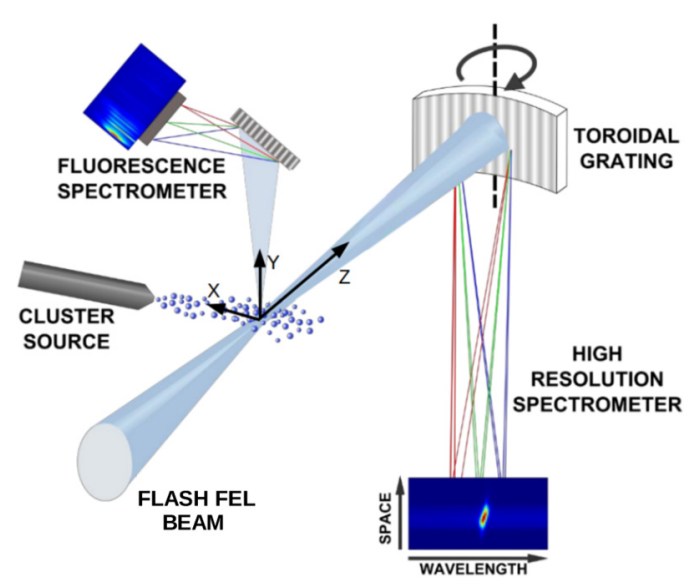

FIG. 1. Scheme of the experiment; see details in the text.

conditions. In Sec. V, the experimental data are discussed in the context of the proposed theoretical model. Conclusions are drawn in Sec. VI. Appendixes A-G give details of the derivations.

\section{EXPERIMENTAL RESULTS}

\section{A. Experimental setup}

The experiment was performed at the CAMP station of the FLASH BL1 beam line [36]. Xe clusters were produced by expansion of a supersonic jet out of a conical nozzle [37]; the details of nozzle geometry are given in Appendix A. Pressure $P_{0}$ and temperature $T_{0}$ in the stagnation chamber behind the nozzle were varied for systematically changing the cluster size distribution and cluster concentration (see Appendix A for details). With employed backing pressures of the cluster source of 0.3-20 bar and gas reservoir temperatures of 220 $290 \mathrm{~K}$, we produced ensembles of clusters with an average radius of $2-25 \mathrm{~nm}$. FEL pulses were focused to a $\sim 10 \times$ $10 \mu \mathrm{m}^{2}$-sized spot onto the interaction point. The interaction of the FEL pulse with the cluster jet results in an elongated, cylindrical-shaped active medium of $\sim 10 \mu \mathrm{m} \times 10 \mu \mathrm{m} \times$ $2 \mathrm{~mm}$ (see a detailed description in Appendix A). We chose FEL photon energies both at the onset $(73 \mathrm{eV})$ and close to the maximum $(92 \mathrm{eV})$ of the giant dipole resonance, resulting in efficient ionization of the Xe $4 d$ electronic shell. In the forward direction, amplified XUV emission at 65 and $68 \mathrm{~nm}$ was dispersed with a setup consisting of a $3 \mathrm{~m}$ focal length toroidal grating and a CCD camera (Andor, model Newton) (see Fig. 1). The grating angle could be varied to select the observable wavelength range, with a typical linear dispersion of $1.7 \mathrm{~nm}$ per 1024 pixels. Note that given the relatively small solid angle of detection of $1.4 \times 10^{-4} \mathrm{sr}$ of this spectrometer, only directed and intense (amplified) lines are observable in the forward direction. In addition to this high-resolution spectrometer, another spectrometer with a larger acceptance angle recorded in the transverse direction the fluorescence emission spectra of the excited clusters in a wider photonenergy range, allowing for time-integrated diagnostics of the ionic charge-state distribution in the cluster [38]. 


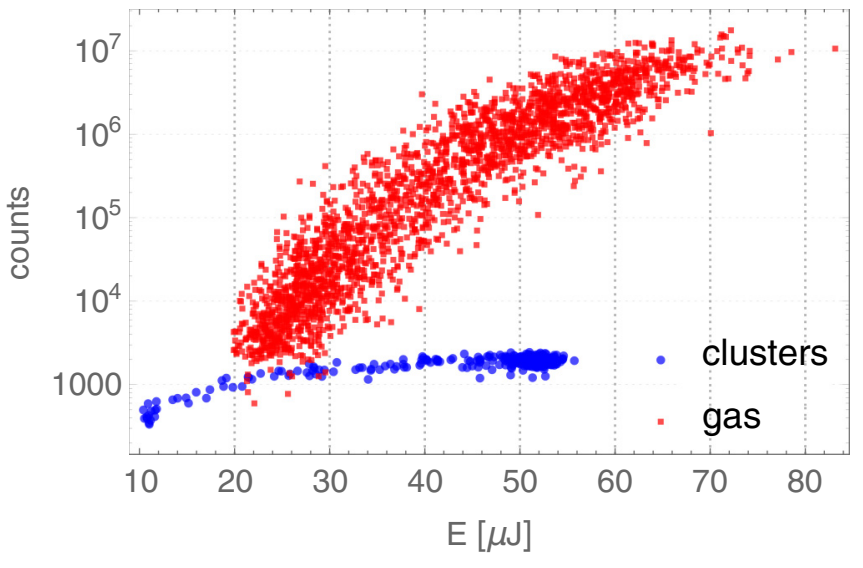

FIG. 2. Comparison of the XUV emission of cluster vs gas phase targets: Detected photon yield of the $65 \mathrm{~nm}$ line as a function of the FEL pulse energy $E$. For the gas target of a pressure of $6.9 \mathrm{mbar}$ (red squares), an exponential signal growth as a function of pump-pulse energy is observed, followed by signs of saturation. In the case of clusters at a stagnation pressure of 5 bar (blue dots) the overall yield is a few orders of magnitude lower. Moreover, only subexponential growth as a function of FEL pulse energy is seen. The FEL photon energy is $73 \mathrm{eV}$ in both cases.

\section{B. Amplified XUV emission: Cluster versus gas target}

We observed two intense emission lines at 65 and $68 \mathrm{~nm}$ emitted in the forward direction. They had been measured and spectrally assigned in a recent gas phase experiment [16], and showed characteristics of superfluorescence. The $65 \mathrm{~nm}$ line was assigned to $\mathrm{Xe}^{2+}$ transitions between configuration-interaction states $0.63\left(5 s^{2} 5 p^{3} 5 d\right)+$ $0.23\left(5 s^{1} 5 p^{5}\right)+0.12\left(5 s^{2} 5 p^{3} 6 d\right)$ and $5 p^{4}$ [39]. As shown by detailed electron-electron coincidence measurements [16], these states are predominantly populated by Auger decay following $4 d$ inner-shell ionisation. We shall see that in the case of Xe clusters, the population of these states for sustaining the amplification of spontaneous emission is due to a different mechanism-electron-ion collisions.

The $65 \mathrm{~nm}$ emission of the cluster target thus shows striking differences from the gas phase case: Fig. 2 compares the emission yield of the $65 \mathrm{~nm}$ line as a function of FEL pulse energy for Xe clusters versus gas. Overall, the emission yield (number of detected photons) of an ensemble of clusters is three to four orders of magnitude smaller than that of a gas phase sample of similar optical density (see Appendix A for a comparison of the number of atoms in both interaction volumes). While an exponential increase of the emission yield as a function of FEL-pulse energy is observable in the gas target, the cluster target only shows subexponential growth. Moreover, the measured emission linewidths are considerably larger in cluster targets (approximately a factor of 10; see also the discussion in Sec. IID) than in the gas phase. (The measurement of the FWHM of the emission in the gas phase was limited by the instrumental broadening). This excludes the collective spontaneous emission of uncondensed Xe atoms, whose fraction is estimated to be less than $20 \%$ [40], as a potential source for stimulated emission. The combination of decreasing emission yield and larger spectral linewidth in a cluster target points to increased decoherence of emitters that hinder superfluorescent emission there. The observed collinear emission stems instead from amplified spontaneous emission. The question here is, how can the harsh cluster environment following FEL interaction sustain amplification at all? To which extent can we, by changing the FEL pulse intensity and hence energy deposition into the cluster, change decoherence and gain in the active cluster medium?

\section{Parameter studies: Intensities, cluster size}

For characterization of the XUV emission of the 65 and $68 \mathrm{~nm}$ lines, we varied the FEL pump-pulse parameters as
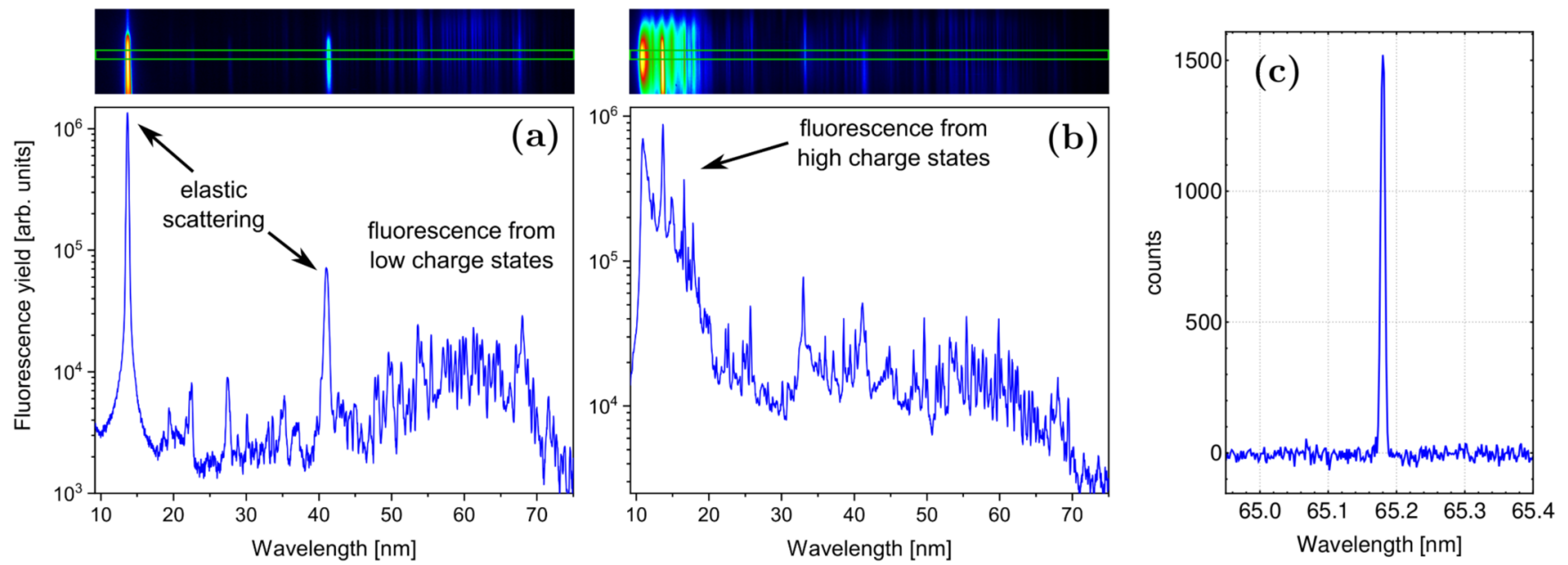

FIG. 3. Typical fluorescence spectra recorded at an FEL pump photon energy of $92 \mathrm{eV}$, a stagnation pressure $P_{0}=10$ bar, and stagnation temperature $T_{0}=290 \mathrm{~K}$ of the cluster source. (a) Raw image of a spectrum recorded in the transverse direction (upper panel) and a spectrum obtained by integrating over the region enclosed by the green rectangle (lower panel). The interaction point is at $z=-9 \mathrm{~mm}$ (shifted from focus), and fluorescence from the low charge states is strong. (b) Similar data to those in (a) for the interaction point positioned at $z=0 \mathrm{~mm}$ (at focus). Fluorescence from high charge states is strong. (c) Spectrum in the forward direction for the interaction point at $z=-9 \mathrm{~mm}$ (shifted from focus). Only the $65.18 \mathrm{~nm}$ line is seen in the observable wavelength range. 


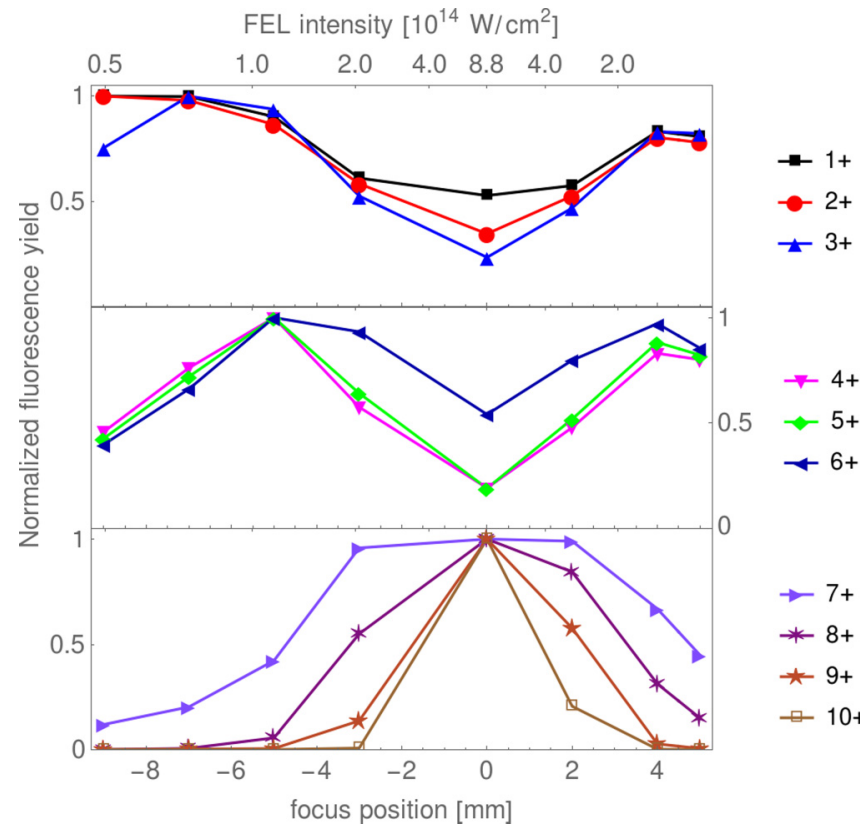

FIG. 4. Variation of charge-state abundance (normalized to the maximal values) vs nozzle displacement from the focus at $z=0$. The upper axis shows estimated values of the mean intensity at the corresponding nozzle position. Pump-photon energy is $92 \mathrm{eV}$, stagnation pressure $P_{0}=5 \mathrm{bar}$, stagnation temperature $T_{0}=290 \mathrm{~K}$, and FEL pulse energy $54 \mu \mathrm{J}$.

well as the cluster-size distribution, and we characterized the (time-integrated) plasma conditions. To change the power density of the FEL, the position of the cluster source (nozzle) was changed along the FEL-beam propagation direction $(z-$ axis in Fig. 1). The analysis of the fluorescence spectra, recorded transversely to the FEL beam propagation, gives insight into the time-averaged plasma conditions: Typical fluorescence spectra are shown in Figs. 3(a) and 3(b). Notably, the intense emission lines of 65 and $68 \mathrm{~nm}$ that are observed in the forward direction [see Fig. 3(c) for a typical signal around the $65 \mathrm{~nm}$ line] could not be found. The line emissions in the region of 10-25 $\mathrm{nm}$ can be assigned to high-charge states $\left(\mathrm{Xe}^{6+\leqslant q \leqslant 11+}\right)$, whereas the fluorescence of the lower charge states appears as characteristic lines in the $45-68 \mathrm{~nm}$ region, allowing for a comparison of relative charge-state abundances at different experimental conditions [41]. Figure 4 shows the normalized ion-charge state abundances as a function of nozzle position; the abundances were obtained in the same way as described in Ref. [41]. Not surprisingly, ions in higher charge states are predominantly produced at the best focus, whereas lower charge states are more abundant in out-of-focus conditions. The high charge states in Xe clusters are partly produced by sequential ionization processes, as well as by electron-impact ionization $[42,43]$. The depletion of the $\mathrm{Xe}^{2+}$ populations has a strong impact on the characteristic XUV emission at 65 and $68 \mathrm{~nm}$ : Fig. 5 shows the emission yield of the $\mathrm{Xe} 65 \mathrm{~nm}$ line in the forward direction as a function of nozzle position. In focus $\left(z=0\right.$, FEL intensity of $\left.\sim 10^{15} \mathrm{~W} / \mathrm{cm}^{2}\right)$, no emission signal was detected. The strongest emission yield was observed for $z=-9 \mathrm{~mm}$ out of focus (FEL intensity of $\sim 5 \times 10^{13} \mathrm{~W} / \mathrm{cm}^{2}$ ).

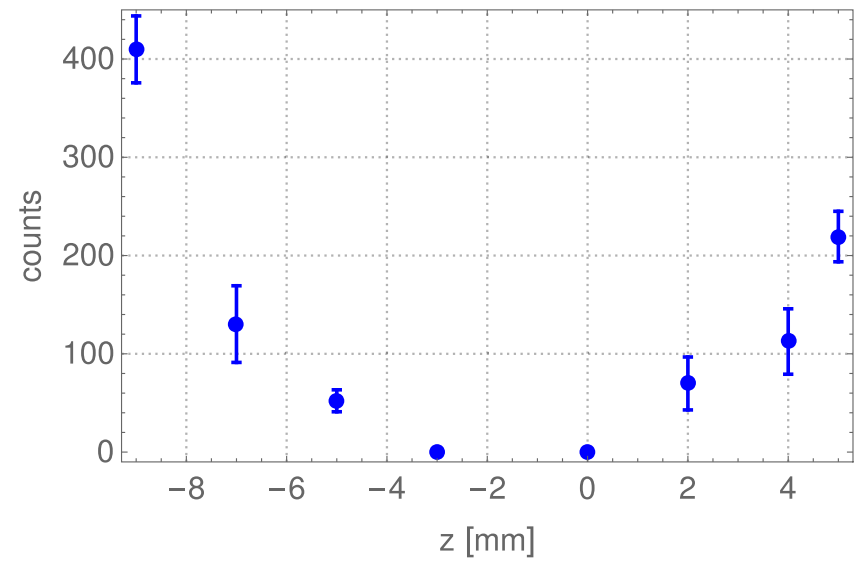

FIG. 5. Dependence of detected photon counts in the forward direction integrated over the $65 \mathrm{~nm}$ line on the position of the cluster jet-FEL interaction point. The $z$ axis is that of the FEL beam propagation (see Fig. 1); $z=0$ corresponds to the assumed focus position. Pump photon energy was $92 \mathrm{eV}$, stagnation pressure $P_{0}=5$ bar, and pump energy $54 \mu \mathrm{J}$.

Additional parameter studies were performed at a nozzle position $z=-9 \mathrm{~mm}$ : Fig. 6 shows the dependence of the $65 \mathrm{~nm}$ line emission yield on the stagnation pressure (a) and stagnation temperature (c) of the cluster source. The signal was detectable from 5 bar stagnation pressure, increased linearly with pressure, and saturation of the emission signal was reached at pressures above 11 bar. Increasing the stagnation pressure results in more atoms in the interaction volume along with a growing cluster radius [44]. Likewise, a decrease in stagnation temperature leads to more atoms in the interaction volume along with also a growing cluster radius [see Eqs. (A1)-(A5) of Appendix A for details], but this behavior is less pronounced than when changing the stagnation pressure, hence also smaller signal variations were recorded. With more atoms in the interaction volume, we observe a narrowing of the emission line-see Fig. 6(b): Together with increasing emission yield, we observe a pronounced decrease of linewidth as a function of pressure for the $92 \mathrm{eV}$ FEL photon energy. This effect, known as "gain-narrowing," is a signature of amplified spontaneous emission [14,15,45]. Figure 7 shows the $65 \mathrm{~nm}$ emission yield and its spectral width (FWHM) as a function of the pump-pulse energy for FEL photon energies of 73 and $92 \mathrm{eV}$. For low pump-pulse energies, the emission yield shows a strong exponential signal increase that saturates at pump-pulse energies of around $20 \mu \mathrm{J}$. Pumping at $92 \mathrm{eV}$ photon energy results in higher energy incoupling and thus higher plasma temperatures, more abundant high charge states, and stronger collision-related decoherence effects. This results in a drop in the amplification gain for $92 \mathrm{eV}$ versus $73 \mathrm{eV}$ and a considerable increase in the linewidth for $92 \mathrm{eV}$ versus $73 \mathrm{eV}$. The actual linewidth for the $73 \mathrm{eV}$ case could be smaller than the $1 \mathrm{meV}$ value observed in Figs. 6(b) and 7, which is at the limit of the spectrometer resolution. Hence, the gain-narrowing effects in Fig. 6(b) and the linewidth dependence on pump energy in Fig. 7 can be obscured by the instrumental effects for the $73 \mathrm{eV}$ case; our 

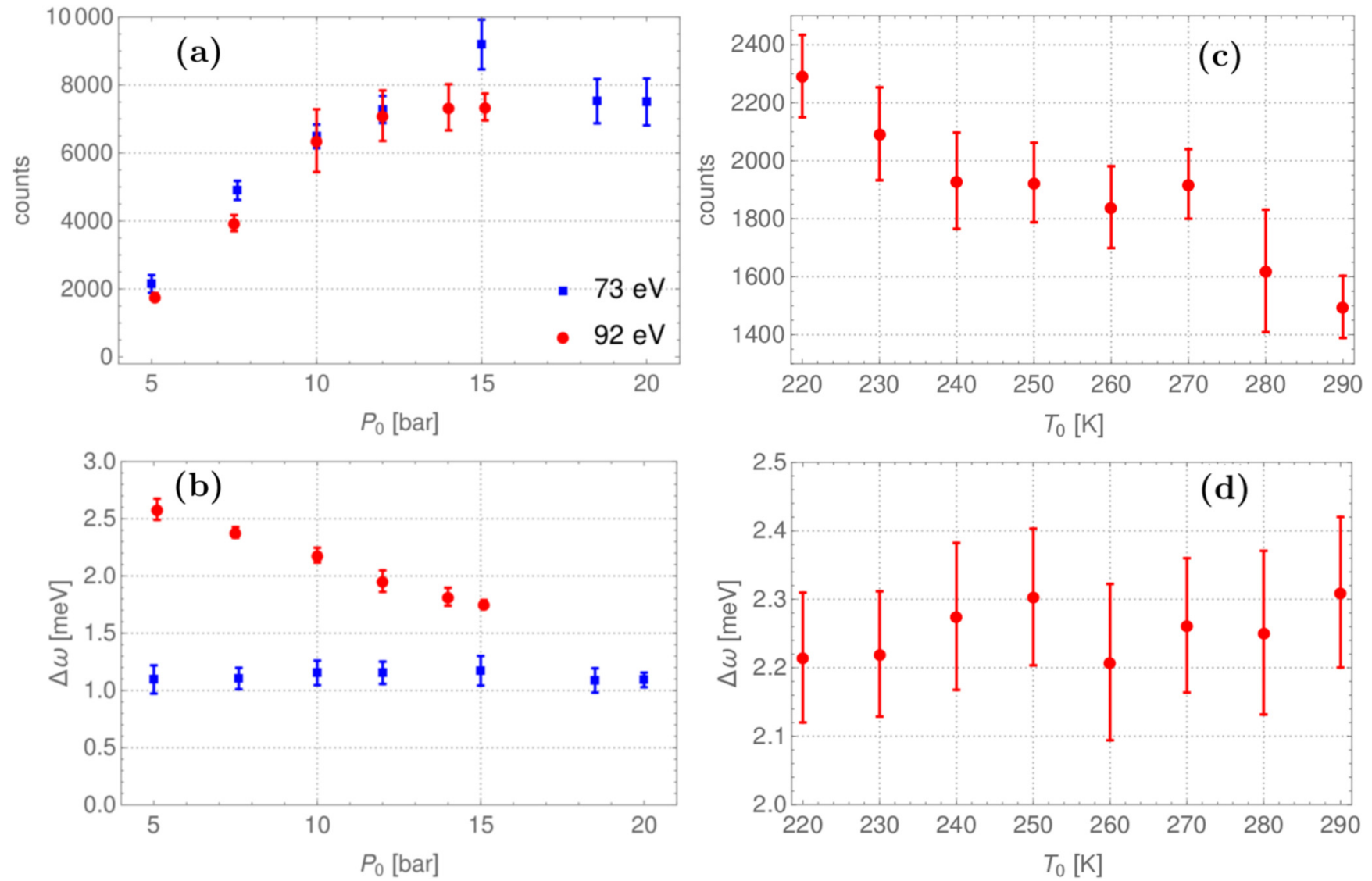

FIG. 6. Detected photon counts (a) and spectral linewidth $\Delta \omega$ (b) of the $65 \mathrm{~nm}$ emission line as a function of the stagnation pressure $P_{0}$ for FEL photon energies of $73 \mathrm{eV}$ (for $52 \mu \mathrm{J}$ pump-pulse energy) and $92 \mathrm{eV}$ (for $47 \mu \mathrm{J}$ pump-pulse energy), stagnation temperature $T_{0}=290 \mathrm{~K}$. Detected photon counts (c) and spectral linewidth $\Delta \omega$ (d) of the $65 \mathrm{~nm}$ emission line as a function of the stagnation temperature $T_{0}$ for $92 \mathrm{eV}$ pump photons, stagnation pressure $P_{0}=5$ bar, and pump energy $47 \mu \mathrm{J}$.

theoretical model supports this assumption (see the end of Sec. V).

\section{Discussion of experimental results}

In our experiments, the typical cluster radius is smaller than the wavelength of the emitted radiation. In the scope of smallsystem superradiance [46], collective spontaneous emission (superfluorescence) of a single cluster (single-cluster superfluorescence) could be the dominant mechanism of radiative decay [see the sketch in Fig. 8(a)]. This collective relaxation seems unlikely, however, since for a collection of atoms in a volume smaller than the radiation wavelength, dipole-dipole interactions cause dephasing, thus quenching superfluorescence $[47,48]$. Moreover, the angular distribution of superfluorescence from a spherical object is isotropic on average [49], contradicting our observation of a directional signal in the forward direction and a missing signal in the transverse direction. Due to the large discrepancy of the wavelengths of the pumping source and the emission line, triggering superfluorescence
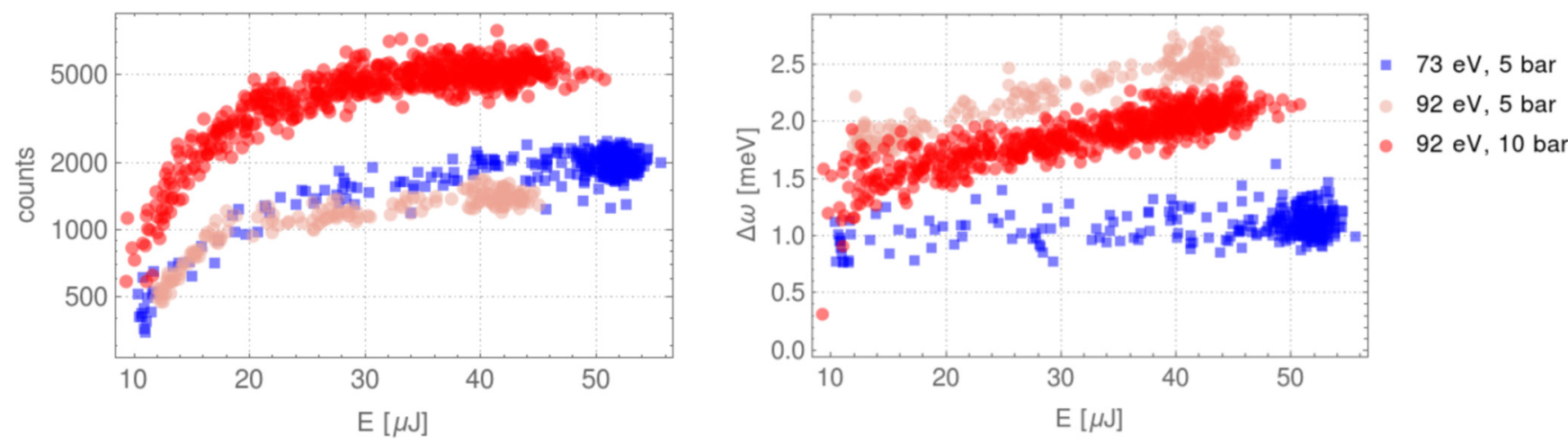

FIG. 7. Dependence of detected photon counts (left) and spectral linewidth $\Delta \omega$ (FWHM, right) on the pump energy $E$. 


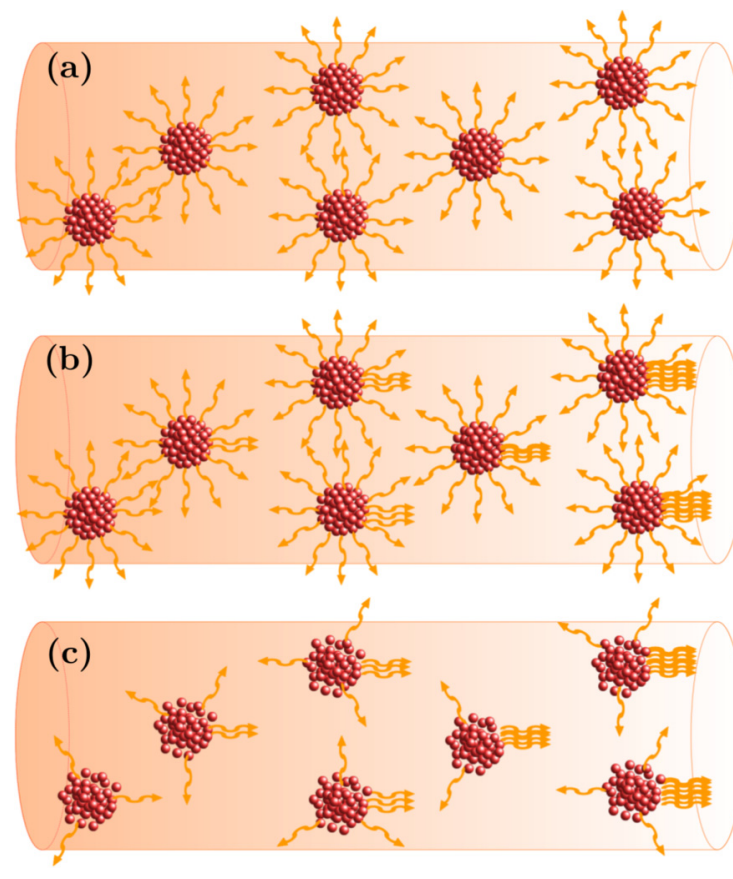

FIG. 8. Sketch of possible scenarios of collective radiation in an ensemble of clusters: (a) single-cluster superfluorescence, (b) intercluster superfluorescence, and (c) amplified spontaneous emission. See the main text for details.

of the clusters by stimulated emission induced by the pump can be excluded. The emission in the forward direction could be reconciled supposing intercluster superfluorescence-a chain reaction in which the superfluorescence from a single cluster stimulates superfluorescence of other clusters in the forward direction [see the sketch in Fig. 8(b)], resulting in exponential signal growth. The amplification in this scenario can easily be estimated. A characteristic quantity determining the enhancement that is due to collective emission is $\frac{3}{8 \pi} n \rho_{\text {inv }} \lambda^{2} l$, where $l$ is the length of the assumed one-dimensional medium, $\lambda$ is the radiation wavelength, $n$ is the concentration of atoms, and $\rho_{\text {inv }}$ is the population inversion $[15,47]$. Independently, the single-cluster superfluorescence is governed by the number of inverted atoms $N_{\mathrm{cl}} \rho_{\mathrm{inv}}$ in the cluster containing $N_{\mathrm{cl}}$ atoms. Therefore, the enhancement that is due to intercluster superfluorescence is determined by $\frac{3}{8 \pi} \frac{n}{N_{\mathrm{cl}}} \lambda^{2} l$, with $\frac{n}{N_{\mathrm{cl}}}$ measuring the number of clusters per unit volume. For a typical experimental case ( $92 \mathrm{eV}$ pump photon energy, $50 \mu \mathrm{J}$ pump energy, 10 bar stagnation pressure, $290 \mathrm{~K}$ stagnation temperature), one obtains at most a factor 3 enhancement over single-cluster superradiance. Hence, our observation that the emission in the forward direction is several orders of magnitude stronger than that in the transverse direction is contradicting the scenario of intercluster superfluorescence and can be excluded as the predominant decay mechanism.

We will show that amplified spontaneous emission (ASE) involving strong decoherence is the predominant decay mechanism, in accordance with all observed experimental characteristics [see the sketch in Fig. 8(c)]. In the WDM environment of FEL-irradiated clusters, electron-ion collisions contribute to decoherence of individual atoms while ion-ion collisions result in dephasing between atoms [50]. As a result, collision- related processes prevent the buildup of a large macroscopic polarization in the medium: a prerequisite for superfluorescence is hence not met. Spontaneous emission in the forward direction can nevertheless stimulate emission of neighboring atoms, resulting in exponential signal growth as a function of target length. Because decoherence of individual atoms and dephasing between atoms hinder the buildup of macroscopic polarization, the associated gain is considerably smaller than in the superfluorescent case. Collisions, however, play also an additional role: Three-body recombination and collisional excitation repopulate the involved electronic states and sustain amplification for a time period of several ps. We substantiate our claim by a quantitative model that compares well with the experimental findings. It takes into account the time evolution of the nanoplasma due to the FEL-cluster interaction, along with a recently developed theory describing superfluorescence and ASE on the same footing.

\section{SIMULATIONS OF CLUSTER EVOLUTION}

ASE in inverted media is a well-known phenomenon in the visible wavelength regime [51-53] and well understood for stationary, homogeneous systems. ASE is the working process of table-top single-pass soft x-ray laser amplifiers [20,54]. This community developed advanced methods to simulate the temporal evolution of the plasma and the amplification process [55]. Here, we aim for the simpler approach of a two-step description of the process that is valid for amplification below the onset of saturation. First, we describe the evolution of the nanoplasma produced by FEL irradiation of the clusters. This will provide the input parameters such as populations of relevant electronic states, etc., for our recently developed method to describe superfluorescence and ASE on equal footing [15].

Even without taking into account the action of the emitted, amplified radiation field, modeling the evolution of atomic population in clusters irradiated by a short intense XUV pulse is a formidable task $[7,43,56-61]$. In the present case, the clusters have a radius of $\sim 15 \mathrm{~nm}$ and contain $\sim 3 \times 10^{5}$ atoms, which renders computations based on an atomistic description such as molecular-dynamics approaches [7,43,5961] too costly. Here, we rely instead on the combination of an atomic kinetic code with a one-dimensional hydrodynamic code in spherical symmetry in order to achieve a macroscopic description of the evolution of ionic mass density, velocity, and electron as well as ion internal energy $[62,63]$. The energy transfer between the electron and the ion subsystem is described by a collision frequency that is interpolated between the Spitzer equation value and the electron-phonon collision frequency, and is limited by a cutoff frequency. The electron internal energy has as a source the power deposited by the FEL into the atomic subsystem and is carried out of the cluster by the emitted electrons. The description of the atomic (ionic) subsystem is done in the configurationaverage (CA) collisional-radiative model [64]. Configurations included in the calculations consist of ground and singly excited configurations (with excitation up to $n=6, n$ being the principal quantum number) from neutral $\mathrm{Xe}$ up to $\mathrm{Xe}^{5+}$. To take into account the photoionization by the FEL, this list of configurations was extended. Additional configurations 
were constructed from all configurations in the list by removing one electron from any outer-valence shell down to $4 d$. For ion charge states above $\mathrm{Xe}^{5+}$, we used a slightly reduced configuration set. For the present experimental conditions, this list of configurations was numerically shown to be sufficient. To include the population dynamics of the characteristic $\mathrm{Xe}^{2+}$ states involved in amplification, states beyond the CA model have been taken into account. In line with the hydrodynamic evolution, we included a four-level model describing the population of the predominant $\mathrm{Xe}^{2+}$ (states that are initially populated by the $4 d$ Auger decay) consisting of the following: the ground state of neutral Xe, the $4 d$ ionized (core-excited) level $[\mathrm{Kr}] 4 d^{9} 5 s^{2} 5 p^{6}$ of $\mathrm{Xe}^{+}$, the ground level $g[\mathrm{Kr}] 4 d^{10} 5 s^{2} 5 p^{4}$ of $\mathrm{Xe}^{2+}$, and the wellidentified excited level $e$ (mixed by configuration interaction) of the kind $0.63[\mathrm{Kr}] 4 d^{10} 5 s^{2} 5 p^{3} 5 d+0.23[\mathrm{Kr}] 4 d^{10} 5 s 5 p^{5}+$ $0.12[\mathrm{Kr}] 4 d^{10} 5 s^{2} 5 p^{3} 6 d$. The two latter levels are populated by Auger decay from the $4 d$ photoionized levels of $\mathrm{Xe}^{+}$, resulting in the population inversion responsible for the $65 \mathrm{~nm}$ emission. The resulting rate equations are

$$
\begin{aligned}
\frac{d p_{0}}{d t} & =-\sigma_{P} j(t) p_{0}+\left(\frac{d p_{0}}{d t}\right)_{\text {coll }}, \\
\frac{d p_{c}}{d t} & =\sigma_{P} j(t) p_{0}-\gamma_{A} p_{c}-\sigma_{P} j(t) p_{c}+\left(\frac{d p_{c}}{d t}\right)_{\text {coll }}, \\
\frac{d p_{e}}{d t} & =b_{e} \gamma_{A} p_{c}-\sigma_{P} j(t) p_{e}+\left(\frac{d p_{e}}{d t}\right)_{\text {coll }}, \\
\frac{d p_{g}}{d t} & =b_{g} \gamma_{A} p_{c}-\sigma_{P} j(t) p_{g}+\left(\frac{d p_{g}}{d t}\right)_{\text {coll }}, \\
p_{0}(0) & =1, \quad p_{c}(0)=p_{e}(0)=p_{g}(0)=0 .
\end{aligned}
$$

Here $\sigma_{P}$ is the ionization cross section, which is assumed to be approximately equal for ground, core-excited, and Auger pumped states [65]; $\gamma_{A}$ is the Auger rate, $b_{e}, b_{g}$ are Auger branching ratios; $j(t)$ is the pump photon flux; and $\left(\frac{d p_{0, c, e, g}}{d t}\right)_{\text {coll }}$ are source/loss terms that are due to collisional and other radiative couplings to all other configurations of the collisionalradiative model. The rates are self-consistently determined based on the evolving plasma parameters. For example, for the excited level $e$ the source/loss term is the following:

$$
\begin{aligned}
\left(\frac{d p_{e}}{d t}\right)_{\text {coll }}= & -\sum_{c^{\prime}}\left(n _ { e } \left[\mathbb{T}_{e c^{\prime}}^{(\mathrm{CE})}\left(T_{e}\right)+\mathbb{T}_{e c^{\prime}}^{(\mathrm{Cl})}\left(T_{e}\right)+\mathbb{T}_{e c^{\prime}}^{(\mathrm{CD})}\left(T_{e}\right)\right.\right. \\
& \left.\left.+\mathbb{T}_{e c^{\prime}}^{(\mathrm{EC})}\left(T_{e}\right)\right]+n_{e}^{2} \mathbb{T}_{e c^{\prime}}^{(\mathrm{CR})}\left(T_{e}\right)\right) p_{e} \\
& +\sum_{c^{\prime}}\left(\mathbb{T}_{c^{\prime} e}^{(\mathrm{A})}+n_{e}\left[\mathbb{T}_{c^{\prime} e}^{(\mathrm{CE})}\left(T_{e}\right)+\mathbb{T}_{c^{\prime} e}^{(\mathrm{Cl})}\left(T_{e}\right)\right.\right. \\
& \left.\left.+\mathbb{T}_{c^{\prime} e}^{(\mathrm{CD})}\left(T_{e}\right)+\mathbb{T}_{c^{\prime} e}^{(\mathrm{EC})}\left(T_{e}\right)\right]+n_{e}^{2} \mathbb{T}_{c^{\prime} e}^{(\mathrm{CR})}\left(T_{e}\right)\right) N_{c^{\prime}}
\end{aligned}
$$

Here, the sum over $c^{\prime}$ is taken over all configurations used in the model; $\mathbb{T}_{e c^{\prime}}^{(\mathrm{CE}, \mathrm{CD}, \mathrm{EC}, \mathrm{CR})}$ (and $\mathbb{T}_{c^{\prime} e}^{(\mathrm{A}, \mathrm{CE}, \mathrm{CD}, \mathrm{EC}, \mathrm{CR})}$ ) are the corresponding rates describing the transition from level $e$ to configuration $c^{\prime}$ (and from configuration $c^{\prime}$ to level $e$ ) by autoionization (A), collisional excitation (CE), collisional ionization $(\mathrm{CI})$, collisional deexcitation $(\mathrm{CD})$, collisional recombination (CR), and electronic capture (CE), respectively; $n_{e}$ and $T_{e}$ denotes the concentration and temperature of plasma electrons; and $N_{c^{\prime}}$ is the population density of configuration $c^{\prime}$. The explicit expressions of rates $\mathbb{T}$ and their dependence on electron temperature can be found in Ref. [64]. For a consistent description of this detailed four-level scheme, within a CA description of the atomic kinetics, the populations of the four-level system are normalized with respect to the populations of the larger collisional-radiative model. Further details on the coupling between the configuration-average collisional-radiative model and the four-level scheme are discussed in Appendix F.

An example for the typical evolution of the macroscopic cluster parameters (at $92 \mathrm{eV}$ photon energy, averaged over the cluster radius) is shown in Fig. 9: The electron temperature rises to $10-50 \mathrm{eV}$ upon interaction with the FEL pulse, reaching its maximum slightly after the maximum of the FEL pulse. An essential contribution to electron heating comes from three-body recombination; see Refs. [62,66]. Through collisions, the energy is transferred between the electronic and ionic subsystems. The ion temperatures reach a maximum at $\sim 1$ ps after FEL interaction. Shortly after FEL interaction, the expansion of the cluster sets in, with a faster expansion rate for higher FEL intensities. During the expansion, both electron and ion temperatures drop. This expansive cooling process is faster for higher FEL intensities: Although initial electron temperatures are higher for higher FEL intensity, the electron temperature for higher pump intensity is smaller than for lower pump intensity after $\sim 10 \mathrm{ps}$. During the expansion, the ionic temperature rapidly drops back to the ambient value.

In Fig. 10, we show the temporal evolution of the (radially averaged) charge-state probabilities up to $10+$ for our experimental conditions out of focus [Fig. 10(a)] and in focus [Fig. 10(b)]. In agreement with the measured fluorescence spectra, charge states above $5+$ become predominant after FEL interaction for the cluster jet in focus. Remarkably, charge states of $2+$ become predominant 5 ps after FEL interaction. At out-of-focus conditions, charge states of $3+$ and $4+$ are predominantly excited right after FEL interaction. Collisional three-body recombination during the expansive cooling, however, repopulates the $2+$ charge states, and they are dominant over a long time window. The steady values of charge states are reached by the end of our simulation period of 15 ps.

To qualitatively compare our simulations with the timeintegrated fluorescence measurement and its evaluation in terms of charge-state abundances of Fig. 4, we show timeintegrated ion abundances (over our simulation period of $15 \mathrm{ps)}$ in Fig. 11 for the corresponding experimental pumppulse intensities (FEL pump energy $54 \mu \mathrm{J}$ and focus positions between $z=0$ and $-9 \mathrm{~mm}$ ). For comparison, we normalized the time-integrated values to their maximum, in accordance with the underlying experimental procedure of Fig. 4, and we accounted for the change of interaction volume at different $z$ positions by multiplying with the beam cross section according to Eq. (A2). The numerical results are in qualitative agreement with experiments: the maximal abundance of ions with higher charge states occurs at larger intensities, while lower charge state abundance reaches maximum at lower intensities. As previously discussed [41], higher intensities drastically change the abundance of high charge states above 

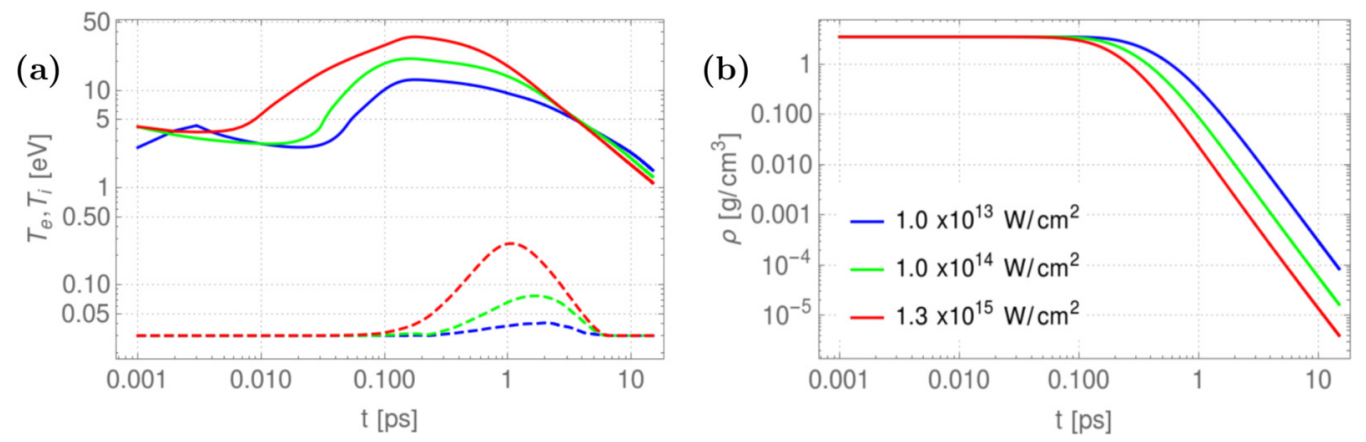

FIG. 9. Time evolution of the nanoplasma: (a) Calculated value of the electron (solid lines) and ion temperatures (dashed lines) of FELirradiated cluster as a function of time. (b) Average ionic density as a function of time. We assumed a cluster radius of $12 \mathrm{~nm}$, an FEL pulse with $92 \mathrm{eV}$ photon energy, and several pump intensities, as indicated in the legend. The temporal profile of the FEL pulse was taken as Gaussian with $80 \mathrm{fs}$ FWHM with its maximum at $t=110 \mathrm{fs}$. The intensity is defined as pulse energy (per $\mathrm{cm}^{2}$ ) divided by FWHM duration.

$9+$, since their production is a result of nonlinear interaction with the FEL pulse. A quantitative comparison would need to account for the radiation-transfer model, and it would require focal averaging and a detailed knowledge of ionic oscillator strengths; this is beyond the scope of this work.

Having understood the dynamics of the overall charge-state distribution, we now focus on the temporal evolution of the occupation $p_{0}$ of the ground state of neutral Xe, occupation $p_{c}$ of the core-excited $4 d^{-1}$ state, and occupations $p_{e}$ and $p_{g}$ of the Auger pumped $\mathrm{Xe}^{2+}$ upper and lower levels (relevant for the emission and amplification of the $\mathrm{Xe}^{2+} 65 \mathrm{~nm}$ line) in Fig. 12. To highlight the importance of electron-ion collisions, we show results of the full model versus simulation results for which all electron-ion collisional processes have been switched off. The difference is striking: In addition to the

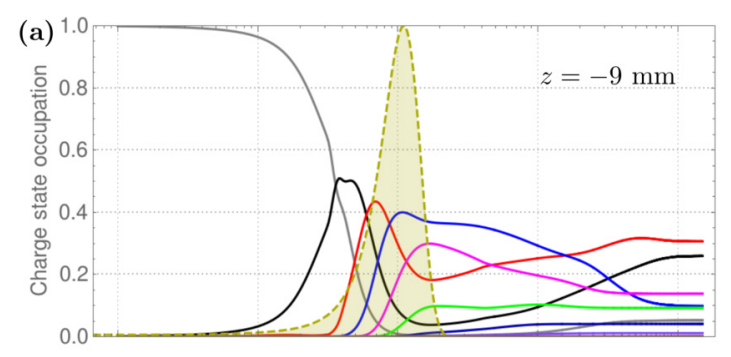

-0
-+1
-+2
-+3
-+4

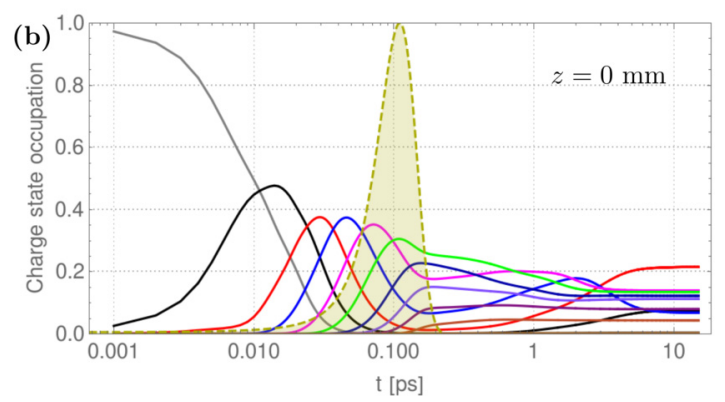

$+5$

$-+6$

$-+7$

$-+8$

$-+9$

$+10$

FIG. 10. Calculated temporal dependence of the cluster average charge state for FEL photon energy of $92 \mathrm{eV}$ and a cluster radius of $12 \mathrm{~nm}$ (corresponding to the mean cluster size at $P_{0}=5$ bar backing pressure, $T_{0}=290 \mathrm{~K}$ backing temperature [see Eq. (A5)]); (a) corresponds to the interaction point position out of focus $(z=$ $-9 \mathrm{~mm})$, (b) corresponds to the position at focus $(z=0 \mathrm{~mm})$. The temporal profile of the pump is shown by a dashed dark green line.
Auger process, collisional ionization in the cluster is a predominant factor for depleting the occupation $p_{c}$ of the $\mathrm{Xe}^{1+}$ core-excited $4 d^{-1}$ state. In the gas phase, the Auger process is the predominant process for creating population inversion; see the discussion in Appendix E. Collisional ionization of the underlying $4 d^{-1}$ state, therefore, results in an initially smaller population inversion. This is directly visible in the populations $p_{e}$ and $p_{g}$ of the relevant $\mathrm{Xe}^{2+}$ states: without collisions, a population inversion between theses states is produced by $4 d$ ionization followed by Auger decay, with a maximum that is reached slightly after the peak of the FEL pulse. Collisional processes, however, completely alter the picture: during the rise time of the FEL pulse, there is actually no population inversion; only slightly after the pulse maximum do collisional processes result in population inversion. In the modeling, the following collisional processes were used [62]: collisional excitation, deexcitation, ionization, and recombination, as well as electronic capture. To understand the relative importance of these processes for establishing the population inversion, a

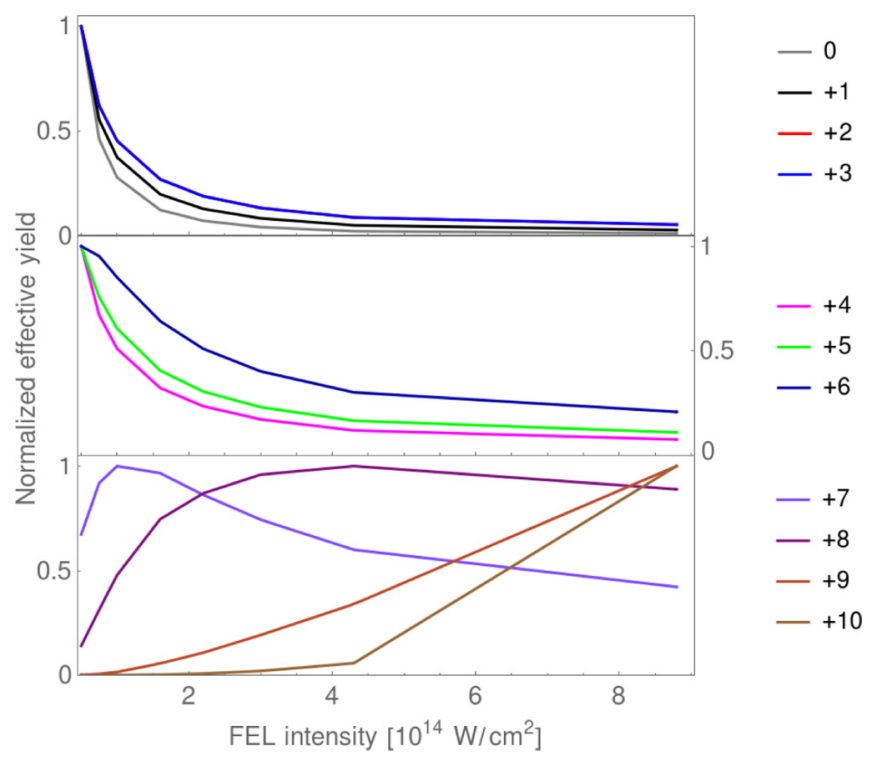

FIG. 11. Temporally integrated and renormalized charge-state probability as a function of FEL intensity (see details in the text). Same parameters as in Fig. 10 are used. 

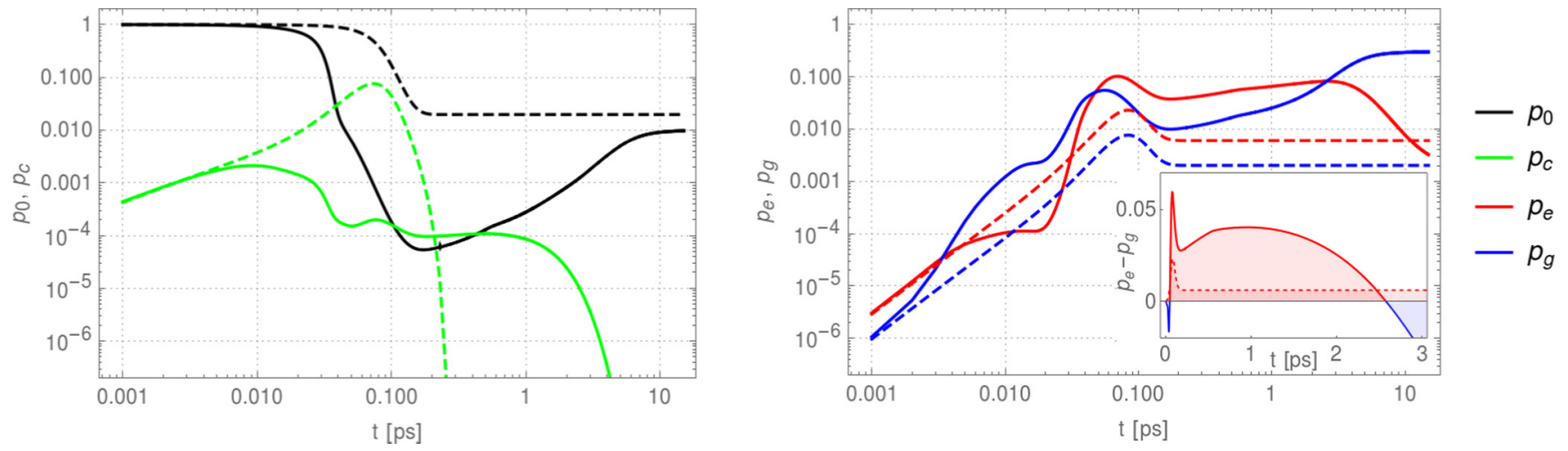

FIG. 12. Temporal evolution of the occupation $p_{0}$ of the Xe ground state, occupation $p_{c}$ of the Xe $\mathrm{e}^{1+}$ core-excited $4 d^{-1}$ state (left), and occupations $p_{e}, p_{g}$ of the Auger pumped $\mathrm{Xe}^{2+}$ upper and lower states (right). The latter show population inversion and are responsible for the $65 \mathrm{~nm}$ ASE. All occupation probabilities have been averaged over the cluster radius. The solid lines correspond to the full model, while the dashed lines show simulations for which all electron-ion collisional processes have been switched off. The inset shows the population inversion in linear scale; positive population inversion is shown in red, negative in blue. We assumed an FEL pump intensity of $3 \times 10^{13} \mathrm{~W} / \mathrm{cm}^{2}$, an FEL-photon energy of $92 \mathrm{eV}$, a pump-pulse duration of $80 \mathrm{fs}$, and a cluster radius of $10 \mathrm{~nm}$.

series of calculations were done where one of the processes was switched off. The results are shown in Fig. 13. The calculations presented in Fig. 13 show that exclusion of any of the collisional processes (except for electronic capture) results in a drastic decrease of the population inversion. Hence, the appearance of high population inversion is a combined effect of collisional excitation, deexcitation, ionization, and three-body recombination in a complex, dynamically evolving plasma. As seen from Eq. (2), the population inversion is influenced by collisional processes, coupling the states $e$ and $g$ to a plethora of different configurations. Each of the collisional processes contributes to the rate in several ways: On the one hand, collisional processes strongly influence the evolution of the concentration $n_{e}$ and temperature $T_{e}$ of

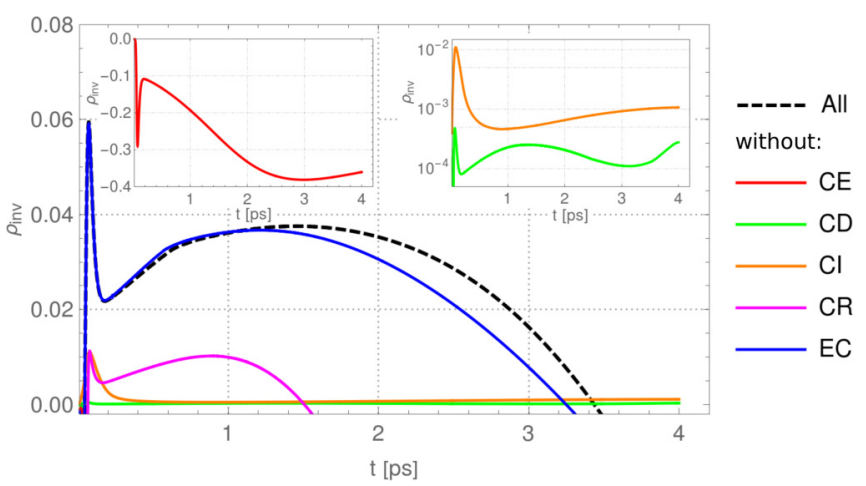

FIG. 13. Role of collisional processes. Temporal evolution of the population inversion (averaged over the cluster) between Xe states that are responsible for the $65 \mathrm{~nm}$ ASE depicted for the following models: full model (black dashed line), full model without one of the following processes: collisional excitation (CE, red, details shown in the left inset), collisional deexcitation ( $\mathrm{CD}$, green, details shown in the right inset), collisional ionization (CI, orange, details shown in the right inset), collisional recombination (CR, magenta), as well as electronic capture (EC, blue). We assumed an FEL pump intensity of $4.2 \times 10^{13} \mathrm{~W} / \mathrm{cm}^{2}$, an FEL-photon energy of $92 \mathrm{eV}$, a pump-pulse duration of $80 \mathrm{fs}$, and a cluster radius of $16 \mathrm{~nm}$. plasma electrons as well as the population density $N_{c}$ of the configurations. According to Eq. (2) (and a similar equation for the population $p_{g}$ of level $g$ ), the parameters $n_{e}, T_{e}, N_{c}$ are crucial ingredients of the source/loss terms, directly and indirectly through a dependence of the rates on $T_{e}$. On the other hand, collisional processes directly contribute to both the loss term [first term in Eq. (2)] and the source term [second term in Eq. (2)] of the level $e$ (and similar for the level $g$ ). Considering the collisional deexcitation process, for example, it produces a source term $\sum_{c^{\prime}} n_{e} \mathbb{T}_{c^{\prime} e}^{(\mathrm{CD})}\left(T_{e}\right) N_{c^{\prime}}$ due to transitions from configurations $c^{\prime}$ (that have energy higher than level $e$ ) to level $e$, and it produces a loss term $-n_{e} \mathbb{T}_{e c^{\prime}}^{(\mathrm{CD})}\left(T_{e}\right) p_{e}$ due to transition from the level $e$ to configurations $c^{\prime}$ that have energy lower than level $e$. Expressions of the same form hold for the level $g$ (with rates $\mathbb{T}_{c^{\prime} g}, \mathbb{T}_{g c^{\prime}}$ calculated for this level). (Note, however, that the loss term would involve the transition to a level belonging to the same configuration-hence, the rate is small and is beyond the accuracy of the configuration-average collisional-radiative model). Similar considerations apply to other collisional processes. As a result, for the population inversion rate $d\left(p_{e}-p_{g}\right) / d t$ it is not straightforward to identify a single dominating collisional process that is responsible for the creation of the population inversion. Importantly, the resulting inversion is more than an order of magnitude higher than without collisions (i.e., pure Auger pumping) and is maintained for a few ps. Thus, the interaction of FEL radiation with xenon clusters results in WDM that sustains ASE in a collisionally excited plasma.

\section{COLLISIONALLY PUMPED XUV AMPLIFICATION}

With a description of the evolution of nanoplasma and the occupation of the relevant states in hand, we apply a recently developed formalism [15] to treat ASE and superfluorescence for transient conditions. The formalism is tailored to determine the $z$-dependent two-time correlation function $G\left(z, \tau_{1}, \tau_{2}\right)$ of the electric field:

$$
G\left(z, \tau_{1}, \tau_{2}\right)=\frac{2 \epsilon_{0} \omega}{\Delta o \hbar c}\left\langle\hat{A}_{-}\left(z, \tau_{1}\right) \hat{A}_{+}\left(z, \tau_{2}\right)\right\rangle .
$$


We assume an effective 1D medium along the direction $z$ of FEL-propagation. $\tau=t-z / c$ is the time with respect to a frame comoving with the FEL-pump pulse. $\omega$ is the transition frequency between excited $e$ and ground $g$ states. $\Delta o$ is the characteristic solid angle into which emission in the forward direction takes place. $\hat{A}_{ \pm}(z, \tau)$ are positive- and negativefrequency parts of the vector potential. The radiated intensity
$I(z, \tau)$ (defined here as the number of photons emitted per solid angle $\Delta o$ per cross section of the system per unit time) is determined by $G(z, \tau, \tau)$. The emission spectrum can be obtained by Fourier transform of $G\left(z, \tau_{1}, \tau_{2}\right)$ according to the Wiener-Khinchin theorem. The propagation of the field correlation function obeys

$$
\begin{aligned}
\frac{\partial G\left(z, \tau_{1}, \tau_{2}\right)}{\partial z}= & -\kappa(z) G\left(z, \tau_{1}, \tau_{2}\right)+g_{1}\left[\int_{0}^{\tau_{1}} d \tau_{1}^{\prime} \mathcal{D}\left(z, \tau_{1}, \tau_{1}^{\prime}\right) \rho_{\mathrm{inv}}\left(z, \tau_{1}^{\prime}\right) G\left(z, \tau_{1}^{\prime}, \tau_{2}\right)+\int_{0}^{\tau_{2}} d \tau_{2}^{\prime} \mathcal{D}\left(z, \tau_{2}, \tau_{2}^{\prime}\right) \rho_{\mathrm{inv}}\left(z, \tau_{2}^{\prime}\right) G\left(z, \tau_{1}, \tau_{2}^{\prime}\right)\right] \\
& +g_{0}\left\{\mathcal{D}\left(z, \tau_{1}, 0\right) \mathcal{D}\left(z, \tau_{2}, 0\right) \rho_{\mathrm{ee}}(z, 0)+\int_{0}^{\min \tau_{1}, \tau_{2}} d \tau^{\prime} \mathcal{D}\left(z, \tau_{1}, \tau^{\prime}\right) \mathcal{D}\left(z, \tau_{2}, \tau^{\prime}\right) s\left(z, \tau^{\prime}\right)\right\},
\end{aligned}
$$

where

$$
\begin{aligned}
g_{1} & =\frac{3}{16 \pi} \Gamma_{\mathrm{sp}} n \lambda^{2}, \quad g_{0}=\frac{3}{32 \pi \lambda^{2}} \Gamma_{\mathrm{sp}} n S_{b} \Delta o, \quad \mathcal{D}\left(z, \tau_{2}, \tau_{1}\right)=e^{-\frac{1}{2} \int_{\tau_{1}}^{\tau_{2}} d \tau^{\prime} \Gamma\left(z, \tau^{\prime}\right)}, \\
\Gamma(z, \tau) & =\Gamma_{\mathrm{sp}}+\gamma_{n}+\gamma_{d}(z, \tau)+\gamma_{e}(z, \tau)+\gamma_{g}(z, \tau), \quad s(z, \tau)=r_{e}(z, \tau)+\left[\gamma_{d}(z, \tau)+\gamma_{g}(z, \tau)\right] \rho_{\mathrm{ee}}(z, \tau) .
\end{aligned}
$$

In Eq. (4), the first term describes the overall absorption with coefficient $\kappa$, and the second term denotes the stimulated emission sustained by the population inversion $\rho_{\text {inv }}$. The third term stands for spontaneous emission with the spontaneous emission rate $\Gamma_{\mathrm{sp}}$, and $S_{b}$ is the geometric cross section of the active medium (the pump-beam cross section). $\Gamma(z, \tau)$ is the total decay rate that determines the linewidth, including the spontaneous decay rate, the nonradiative transition rate $\gamma_{n}$ between excited and ground states, the decoherence rate $\gamma_{d}$, and decay rates of excited $\gamma_{e}$ and ground $\gamma_{g}$ states. $r_{e}$ is the total pumping rate of the excited-state population $\rho_{e e}$. The general expression (4) can be simplified in the case of large decoherence rate, i.e., $\gamma_{d}$ much larger than all other rates present in (5). In this case, it is convenient to express the correlation function as

$$
G_{s}(z, \tau, \Delta \tau):=G(z, \tau-\Delta \tau / 2, \tau+\Delta \tau / 2), \quad G_{s}(z, \tau, \Delta \tau):=\int d \omega e^{i \omega \Delta \tau} G(z, \tau, \omega)
$$

and $G(z, \tau, \omega)$ satisfies the following equation (see Appendix B for the derivation):

$$
\frac{\partial G(z, \tau, \omega)}{\partial z}=\left[g_{1} \rho_{\mathrm{inv}}(z, \tau) \frac{\gamma_{d}(z, \tau)}{\left[\gamma_{d}(z, \tau) / 2\right]^{2}+\omega^{2}}-\kappa(z)\right] G(z, \tau, \omega)+\frac{g_{0}}{2 \pi} \rho_{\mathrm{ee}}(z, \tau) \frac{\gamma_{d}(z, \tau)}{\left[\gamma_{d}(z, \tau) / 2\right]^{2}+\omega^{2}} .
$$

The spectral intensity $I(\omega)$ is obtained by integration of $G(z, \tau, \omega)$ over the emission time $\tau$. Solving Eq. (7), we get

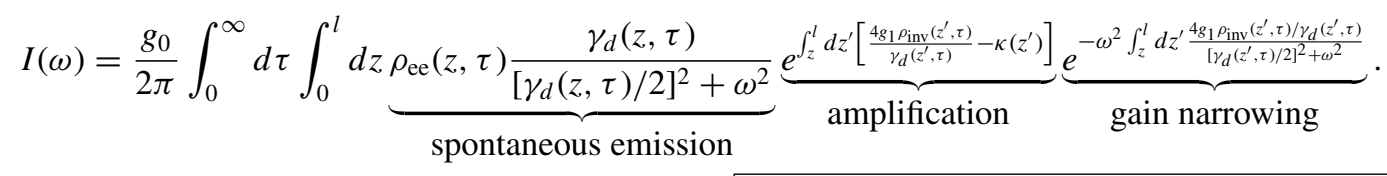

The preexponential factor in Eq. (8) gives the amount of spontaneous emission, the first exponent its amplification, and the second exponent describes the spectral modification by gain narrowing $[51,53]$. The upper limit of the time integral is formally infinity; in practice, we extend the integral only over the time interval for which $\rho_{\text {inv }} \geqslant 0$, neglecting reabsorption. Unsurprisingly, the emitted spectral intensity $I(\omega)$ depends on the temporal and spatial evolution of the excited-state occupation $\rho_{\mathrm{ee}}(z, \tau)$ and the population inversion $\rho_{\mathrm{inv}}(z, \tau)$. In general, their evolution has to be calculated taking into account the interaction with the emitted radiation. This becomes important in the limit of strong superfluorescence and saturation of the amplification, giving rise to the typical temporal ringing behavior and Rabi-like oscillations $[15,47]$. In the present case, far from saturation, the influence of the emitted field on the evolution of the population of atomic levels is negligible (see the estimate in Appendix C). The occupation of the electronic states can thus be assumed unaffected by the emitted field, but generally depends on $z$ and $t$. (For a constant value of the level populations, an analytical expression of the field- correlation function can be derived; see Appendix D). During the relevant emission times, the single cluster expands and the level occupations change as a function of time. For the cluster ensemble, the propagating FEL pulse is heavily absorbed, so that clusters at larger penetration depth $z$ experience lower field intensities and thus different levels of inversion. We treat the absorption of the FEL by a linear absorption coefficient $\kappa_{p}$ (as result, the dependence of cluster evolution on $z$ can be mapped to a dependence on the pump-pulse intensity by $I_{p}(z)=I_{0} e^{-\kappa_{p} z}$ ), assuming "cold absorption," and we neglect saturated absorption and potential changes of the opacity of the medium upon evolution of the plasma. To account for the cluster medium, we treat the FEL absorption by Mie scattering theory (see Appendix G). For the ASE radiation, we neglect absorption in the medium $[\kappa(z)=0]$, since its photon energy of $19 \mathrm{eV}$ lies below the absorption edge of singly and higher charged Xe ions.

To highlight the drastic effects on FEL intensity, Fig. 14 shows the temporal dependence of the population inversion of a single cluster placed at different focus positions along the 


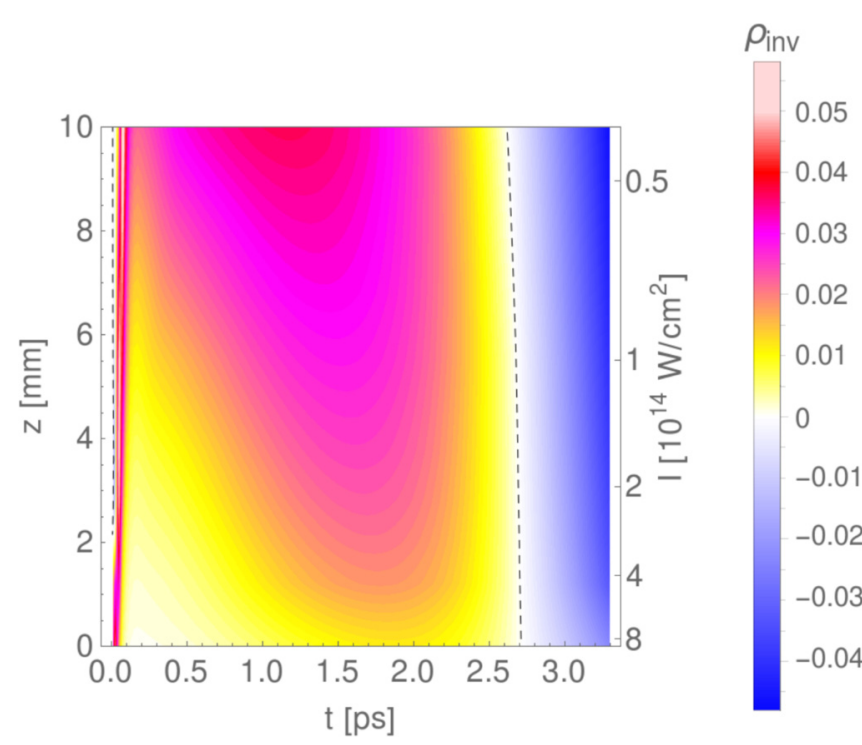

FIG. 14. Temporal evolution of the population inversion $\rho_{\text {inv }}=$ $p_{e}-p_{g}$ as a function of average intensity [corresponding to different cluster-jet positions $z$ with respect to the focus $(z=0)]$. We assumed a pump-pulse energy of $54 \mu \mathrm{J}$ and a focus-size dependence on the distance from the focus according to Eq. (A2). Dashed lines separate the regions of positive and negative population inversion.

FEL propagation axis (in accordance with Fig. 5 discussing the emission yield as a function of cluster-jet position along the propagation direction of the FEL). In the focus $(z=0)$ with intensities approaching $10^{15} \mathrm{~W} / \mathrm{cm}^{2}$, the population inversion is only substantial during the first few tens of fs and is quenched by multiple ionization of the target. For $t \gtrsim 1.5 \mathrm{ps}$, the cooling and expansion of the cluster results in a slight population inversion. Out of focus $(z=10 \mathrm{~mm})$, for which the highest gain is observed, a substantial population inversion builds up after the interaction with the laser pulse, with a duration of roughly $2-3$ ps.

\section{MODELING THE EXPERIMENTAL DATA}

The level of collective radiative emission is strongly influenced by decoherence rate $\gamma_{d}(z, \tau)$ that influences both photon yield and spectral shape; see Eq. (8). The decoherence rate $\gamma_{d}$ is a cumulative parameter introduced to embrace decoherence of individual emitters (e.g., conditioned by dephasing due to collision with electrons $[50,67])$ and dephasing between emitters (e.g., due to random frequency shifts caused by ion microfields [50,67], dipole-dipole interaction $[47,48]$, Doppler effect, etc.). Inclusion of dephasing between emitters (that causes inhomogeneous broadening) into a decoherence parameter (that describes homogeneous broadening) is an acceptable approximation for description of the linear stage of collective emission (to which ASE belongs); see Ref. [68]. Modeling of the decoherence rate is thus a very difficult, if not impossible, task. Moreover, the inhomogeneity of the expanding cluster poses further complications: Fig. 15 shows the evolution of plasma parameters (electron density, electron temperature, population inversion) for different positions within a single cluster according to our numerical model. The cluster center features higher population inversion and dominantly contributes to the ASE. As expected, the outer cluster shells undergo rapid expansion with a rapid drop of the electron density [shown in Fig. 15(c)]. The evolution of the electron temperature is similar for all cluster regions [see Fig. 15(d)]. Within the consideration of local thermodynamic equilibrium, these heterogeneous conditions would lead to a broad variety of spectral emission shapes, even in the limit of independent emission of single atoms. In Fig. 15(e), we show the independent (noncollective) emission profiles of various snapshots in time and space, as calculated by the PPP code (see Ref. [69] for code details) for the $65 \mathrm{~nm}$ line emission. The line shape, and thus a phenomenological decoherence rate that could be defined for a specific transient plasma condition, depend strongly on time and position within the cluster.

Within our model, we try to estimate an effective decoherence rate by comparing the experimentally observed photon yield and spectral width (see Fig. 7) to our calculation. Because of the integral form of Eq. (8), a full reconstruction of $\gamma_{d}(z, \tau)$ by the limited experimental data seems intractable without a priori assumptions, and might per se be an ill-posed inverse problem. Due to the complexity of the decoherence process and the inhomogeneity of the active medium, it is hard to make any justified assumptions about the time and intensity dependence of the decoherence rate. Therefore, we aim for an estimate of an averaged, effective value of $\gamma_{d}(E)$ with a parametric dependence on the applied pump-pulse energy $E$ : For a given cluster size and $E$, we assume the decoherence rate to be constant over time, cluster volume, and propagation distance. For a particular $E$, we evaluate Eq. (8) with calculated level occupancies and adjust $\gamma_{d}$ in the simulation, so that the numerically determined total emission yield or spectral width coincide with their experimental values.

Figure 16(a) shows the estimated decoherence time $t_{d}=$ $1 / \gamma_{d}$ by comparing to the total yield (red curve) and spectral width (blue curve) for the experimental data presented in Fig. 7. The obtained values are in the range 100-200 fsabout one order of magnitude smaller than the duration of the population inversion. This supports the assumption of a large decoherence rate that was taken during the transition from Eq. (4) to Eq. (7). Evidently, the values for the effective decoherence time obtained from photon yield are different from those obtained by comparing the spectral width. A constant value $\gamma_{d}$ thus cannot reproduce both spectral width and total yield. Analysis of Eq. (8) in the limit of strong amplification shows substantial differences in the functional dependence of the leading terms of $\gamma_{d}$ : For strong amplification and gain narrowing, one can neglect the $\omega^{2}$ term in the denominator of Eq. (8), resulting in a purely Gaussian spectrum with the FWHM given by

$$
\Delta \omega_{\mathrm{FWHM}}=\left(\int_{z}^{l} d z^{\prime} \frac{4 g_{1} \rho_{\mathrm{inv}}\left(z^{\prime}, \tau\right)}{\ln (2) \gamma_{d}\left(z^{\prime}, \tau\right)^{3}}\right)^{-\frac{1}{2}} .
$$

The photon number, however, is mostly determined by the argument of the first exponent in Eq. (8) - the effective gainlength product:

$$
g L=\int_{z}^{l} d z^{\prime} \frac{4 g_{1} \rho_{\mathrm{inv}}\left(z^{\prime}, \tau\right)}{\gamma_{d}\left(z^{\prime}, \tau\right)} .
$$



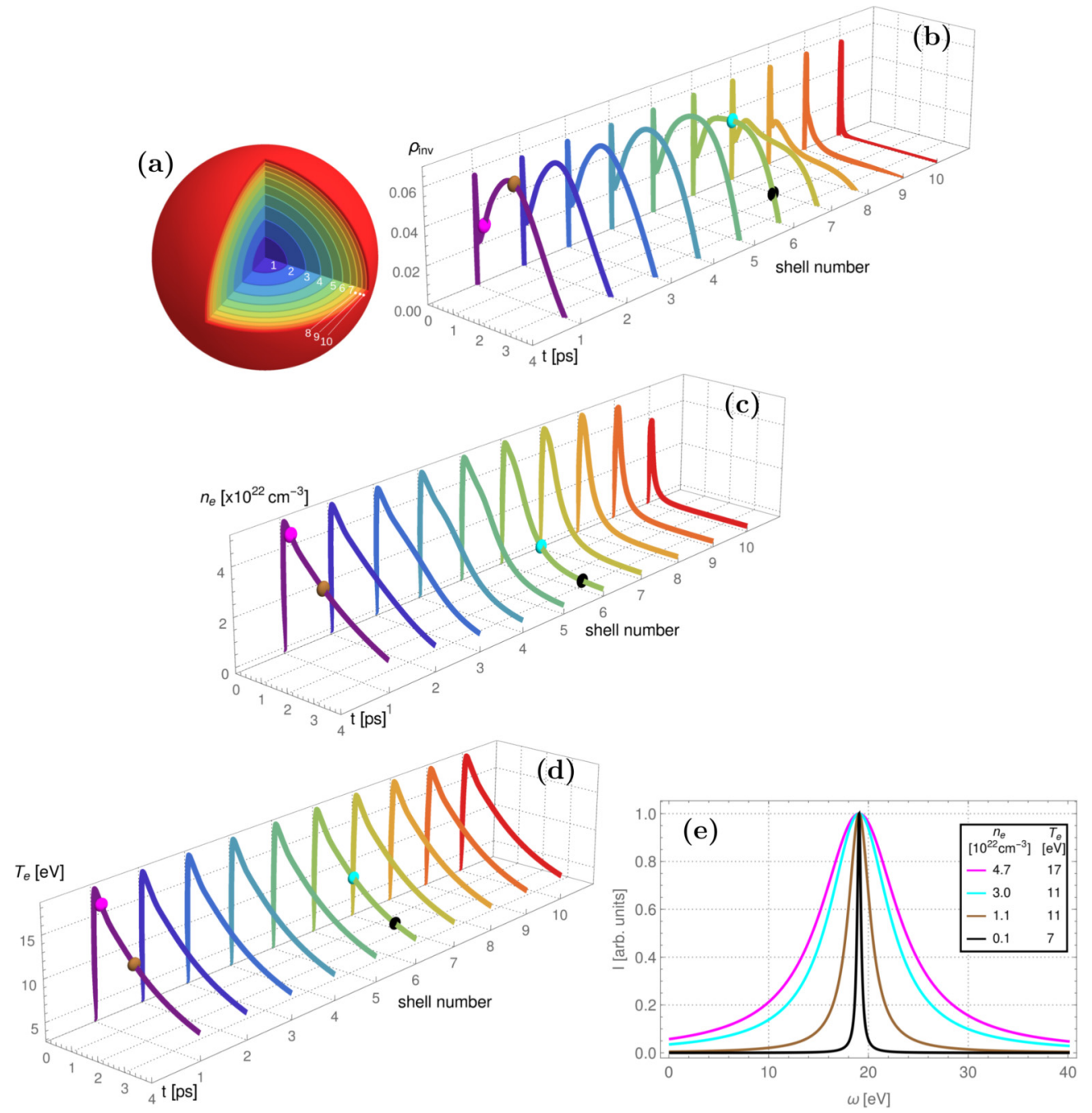

FIG. 15. Simulation of the temporal evolution of various plasma parameters of a single cluster of $16 \mathrm{~nm}$ radius subject to an FEL intensity of $4.2 \times 10^{13} \mathrm{~W} / \mathrm{cm}^{2}$, a pulse duration of $80 \mathrm{fs}$, and a photon energy of $92 \mathrm{eV}$ : (a) Sketch of discretization of the cluster into shells, defining the color code for (b)-(d). The simulation is done in 10 shells with a width following a geometric progression with a common ratio of 0.8 . The mass of each cell remains constant over the temporal evolution (Lagrangian description). (b) Population inversion $\rho_{\text {inv }}$. (c) Electron density $n_{e}$. (d) Electron temperature $T_{e}$. (e) Calculated line shapes for single-atom emissions for several time-space snapshots [marked by dots of the same color in (b)-(d)]: magenta color corresponds to $n_{e}=4.7 \times 10^{22} \mathrm{~cm}^{-3}, T_{e}=17 \mathrm{eV}$, resulting FWHM is $\Delta \omega=9.4 \mathrm{eV}$, cyan corresponds to $n_{e}=3.0 \times 10^{22} \mathrm{~cm}^{-3}, T_{e}=11 \mathrm{eV}, \Delta \omega=7.3 \mathrm{eV}$, brown corresponds to $n_{e}=1.1 \times 10^{22} \mathrm{~cm}^{-3}, T_{e}=11 \mathrm{eV}, \Delta \omega=2.6 \mathrm{eV}$, black corresponds to $n_{e}=0.1 \times 10^{22} \mathrm{~cm}^{-3}, T_{e}=7 \mathrm{eV}, \Delta \omega=0.45 \mathrm{eV}$.

Evidently, parameters (9) and (10) have a different functional dependence on $\gamma_{d}(z, \tau)$. For fitting both emission yield and width, it would be necessary to develop a functional expression for $\gamma_{d}(z, \tau)$.

Despite the simplicity of the assumed constant decoherence rate, it helps in understanding the influence of the gain-narrowing effect on the observed linewidth. Figure 6(b) shows that for $92 \mathrm{eV}$ pump-photon energy, the linewidth decreases with increasing stagnation pressure (implying an increase in density). A "naive" estimate of the decoherence time according to $\hbar / \Delta \omega_{\mathrm{FWHM}}$ would result in an increase of the decoherence time with increasing stagnation pressure. On the contrary, an estimate taking into account ASE effects shows the opposite trend. Accordingly, Fig. 17 shows the estimates of the decoherence time versus stagnation pressure based on Eq. (8). Here, population inversion and excited-state populations were calculated for clusters having a radius that depends on stagnation pressure $P_{0}$ and temperature $T_{0}$ according to Eq. (A6), and the decoherence rate was assumed to be constant [the same procedure of estimating as for Fig. 16(b) was used]. The obtained decoherence time shows almost no dependence on the stagnation pressure and is about three times shorter than the corresponding value from the "naive" estimate. This difference, as well as the experimentally observed trend, can be explained based on the phenomenon of gainnarrowing. An increase of the stagnation pressure results in a higher concentration of Xe atoms [see Eq. (A1)] and hence in a higher optical density-length product of the amplifying medium. According to Eq. (9), this leads to a decrease of the linewidth, in agreement with the trend observed in Fig. 6(b). In the case of $73 \mathrm{eV}$ pump-photon energy, the gain-narrowing should take place as well, however it could not be detected 

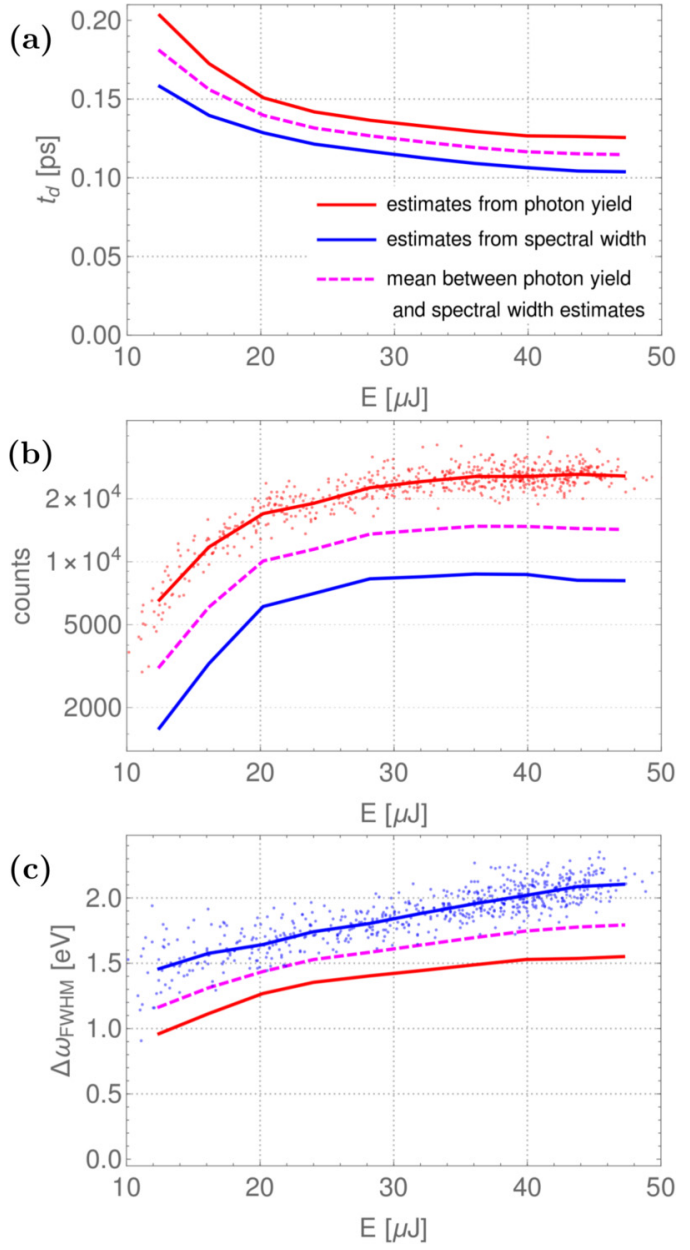

FIG. 16. (a) Estimates of the decoherence time from the photon yield (red line), spectral width (blue line), and mean between them (dashed magenta line) corresponding to the experimental data presented in Fig. 7 by red dots (photon energy $92 \mathrm{eV}$, stagnation pressure $P_{0}=10 \mathrm{bar}$, and stagnation temperature $T_{0}=290 \mathrm{~K}$ ). (b) Experimental dependence of photon yield on pump energy (from Fig. 7) compared to the simulation results corresponding to a fixed decoherence rate. Red line, decoherence rate was estimated from photon yield; blue line, from spectral width; dashed magenta line, mean between them. (c) Same as (b) for spectral width experimental data, and corresponding simulations.

due to instrumental broadening, which is about $1 \mathrm{meV}$. The decoherence time and corresponding expected linewidth can be estimated based on the photon yield (similar to the estimates presented by red lines in Fig. 16). For example, for 5 bar stagnation pressure, one obtains decoherence time $t_{d}=0.3 \mathrm{ps}$ and corresponding linewidth $\Delta \omega_{\mathrm{FWHM}}=0.6 \mathrm{meV}$, which is below our instrumental resolution.

\section{CONCLUSIONS}

Submitting a jet of nanometer-sized Xe clusters to intense pulses of a soft X-ray free-electron laser results in an expanding nanoplasma of WDM that shows large amplification gain on transitions in the XUV spectral region. We presented a combined experimental and theoretical study on the amplified spontaneous emission of the $\mathrm{Xe}^{2+} 65 \mathrm{~nm}$ line transition in

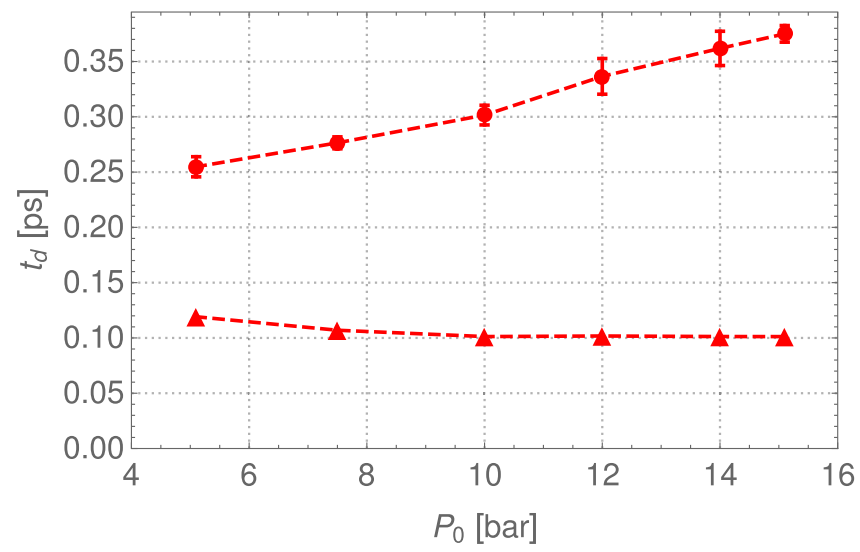

FIG. 17. Gain narrowing demonstration: decoherence time estimates from linewidth dependence on stagnation pressure for the case of $92 \mathrm{eV}$ pump photon energy, and $47 \mu \mathrm{J}$ pump pulse energy [data are shown in Fig. 6(b)]. Circles with error bars show the decoherence time estimated according to $\hbar / \Delta \omega_{\mathrm{FWHM}}$; triangles show estimates of decoherence time according to the same procedure as was described in the text and used for the simulations shown in Fig. 16. Dashed red lines are guides to the eye.

a pencil-shaped active medium of Xe clusters, following the interaction of FEL pulses tuned above the $4 d$-ionization edge of $\mathrm{Xe}$. In the gas phase, $4 d$ ionization and a subsequent Auger decay results in a long-lived population inversion and the emission of superfluorescent high-intensity XUV radiation [16], near the maximum cross section of neutral xenon at the giant resonance. In the warm-dense nanoplasma environment, collisions are crucial and have twofold influence. First, collisions result in strong decoherence, so that at the considered optical densities the collective XUV emission can be characterized as amplified spontaneous emission. Experimentally, we observe an increase in the emission linewidth as compared to the gas phase experiments and exponential amplification gain at lower emission yields than in the gas phase sample. For constant FEL pump-pulse energy, the emission linewidth decreases with increasing optical density (stagnation pressure). This effect, known as gain narrowing, is a clear indication of amplified spontaneous emission. Second, collisions modify strongly the formation of population inversion. The relatively high photon yields can only be explained by an extended time duration during which population inversion exists. Comparing experimental results to our theoretical model, which accounts for the spatial and temporal evolution of the cluster, the treatment of radiation transfer, and collective amplification, we conclude that collisions in the nanoplasma are critical to build up and sustain population inversion in the system. The resulting population inversion depends strongly on the FEL pulse intensity. In terms of maximizing ASE yield, the optimal value of FEL pulse intensity (about $\sim 5 \times$ $10^{13} \mathrm{~W} / \mathrm{cm}^{2}$ ) is a result of the tradeoff between enhancing processes that lead to the population inversion and avoiding the multiple ionization of the target leading to an entirely different plasma state of higher charge states and temperature. In comparison to the gas phase, we infer inversion densities that are $\sim 10$ times higher and a duration of the population inversion of $\sim 2 \mathrm{ps}$, in spite of strong decoherence. By 
comparing our theory to the experimental data, we estimate typical decoherence times of approximately 100-200 fs.

The amplified spontaneous emission signal is formed while the population inversion is present in the system-in our case the first few ps after irradiation with the FEL pulse. It can hence be considered as a time-gated signal. Unlike fluorescence spectroscopy or time-of-flight mass spectroscopy, which yield time-averaged information of the evolving nanoplasma, ASE can give insight into the plasma properties within a tight observation time window.

\section{ACKNOWLEDGMENTS}

The authors gratefully acknowledge the work of the scientific and technical team at FLASH, who made these experiments possible. We acknowledge the Max Planck Society for funding the development and the initial operation of the CAMP end-station within the Max Planck Advanced Study Group at CFEL and for providing this equipment for CAMP@FLASH. The installation of CAMP@FLASH was partially funded by the BMBF Grants No. 05K10KT2, 05K13KT2, 05K16KT3, and 05K10KTB from FSP-302. D.Ro. acknowledges support from the Chemical Sciences, Geosciences, and Biosciences Division, Office of Basic Energy Sciences, Office of Science, US Department of Energy, Grant No. DE-FG02-86ER13491. C.B. B.E., and D.Ro. were also supported by the Helmholtz Gemeinschaft through a Helmholtz Young Investigator grant to D.Ro. T.M., D.Ru., B.L., A.N., and M.M. acknowledge support by the Deutsche Forschungsgemeinschaft under Grants No. MO 719/13-1, MO 719/14-1, and by the Bundesministerium für Bildung und Forschung (BMBF, project ID 05K16KT3). T.L. acknowledges support from the Deutsche Forschungsgemeinschaft through the Cluster of Excellence "Advanced Imaging of Matter" (EXC 2056-project ID 390715994).

A.B. and L.M. equal contribution to the work.

\section{APPENDIX A: MODELING OF THE CLUSTER JET}

The gas concentration $n$ at the distance $x_{\mathrm{IP}}$ from the conical nozzle orifice is given by [44]

$$
\begin{aligned}
n & =\frac{P_{0}}{k_{B} T_{0}} \frac{0.15}{\delta^{2}}, \\
\delta & =\frac{x_{\mathrm{IP}}}{d_{\mathrm{eq}}}=\frac{l}{2 d_{\mathrm{eq}} \tan \alpha}, \\
d_{\mathrm{eq}} & =0.74 \frac{d_{\text {orifice }}}{\tan \alpha},
\end{aligned}
$$

where $P_{0}$ is the pressure in the gas reservoir before the nozzle (stagnation pressure), $T_{0}$ is the stagnation temperature, $l$ is the diameter of the jet at the interaction point, $d_{\mathrm{eq}}$ is the equivalent orifice diameter, and $\alpha$ is the half opening angle of the nozzle cone (for our case $\alpha=4 \mathrm{deg}, d_{\text {orifice }}=200 \mu \mathrm{m}$ ). The diameter of the jet at the interaction point $l$ was obtained from a scan of radiative emission in the forward direction versus transverse position (height) of the cluster source. It comes out to be about $2.1 \mathrm{~mm}$. From Eq. (A1) we obtain that the distance of the nozzle from the interaction point is $x_{\mathrm{IP}}=15 \mathrm{~mm}$. The shape of the interaction volume, determined by the overlap of the
FEL focal volume and the cluster jet, is pencil-like, the length is given by $l$, and the cross section is given by the size of the FEL beam. Depending on the position $z_{\mathrm{IP}}$ of the interaction point with respect to the FEL focus (along the FEL beam propagation), the size of the FEL beam can be estimated from the data provided in Ref. [36], Fig. 3. Fitting the results of PMMA imprint beam size measurement [36], one obtains

$$
S\left(z_{\mathrm{IP}}\right)=\left(8.1+2.9 z_{\mathrm{IP}}[\mathrm{mm}]\right) \mu \mathrm{m} \times\left(9.4+3.4 z_{\mathrm{IP}}[\mathrm{mm}]\right) \mu \mathrm{m} .
$$

According to the semiempirical scaling laws established by Hagena [70], the condensation process can be characterized by the scaling parameter [71]

$$
\Gamma^{*}=5554[\text { for Xe }] \frac{\left(d_{e q}[\mu \mathrm{m}]\right)^{0.85} P_{0}[\mathrm{mbar}]}{\left(T_{0}[\mathrm{~K}]\right)^{2.29}} .
$$

The mean number of atoms within one cluster $\left(N_{\mathrm{cl}}\right)$ is connected to $\Gamma^{*}$ by empirical relations $[37,72,73]$ :

$$
\begin{aligned}
\text { for } \Gamma^{*}<350: & N_{\mathrm{cl}}=1, \\
\text { for } 350 \leqslant \Gamma^{*}<1800: & N_{\mathrm{cl}}=38.4\left(\frac{\Gamma^{*}}{1000}\right)^{1.64}, \\
\text { for } \Gamma^{*} \geqslant 1800: & N_{\mathrm{cl}}=33\left(\frac{\Gamma^{*}}{1000}\right)^{2.35} .
\end{aligned}
$$

In our case, the clusters are relatively large, i.e., $\Gamma^{*}>10000$, and the number of atoms is estimated as $[74,75]$

$$
\text { for } \quad \Gamma^{*} \geqslant 10000: \quad N_{\mathrm{cl}}=100\left(\frac{\Gamma^{*}}{1000}\right)^{1.8} .
$$

The cluster radius is connected to $N_{\mathrm{cl}}$ as

$$
r_{\mathrm{cl}}=r_{s} N_{\mathrm{cl}}^{1 / 3}
$$

where $r_{s}$ is Wigner-Seitz radius (for Xe, $r_{s}=2.45 \AA$ [42]).

\section{Estimates of the number of emitters}

The concentration of atoms in the cluster jet at a given stagnation pressure corresponds to the concentration in the gas phase for an equivalent pressure given by $P_{\text {equivalent }}=$ $P_{0} 0.15 / \delta^{2}$. In the case of the Xe gas experiment (data presented in Fig. 2), the length of the pencil-shaped interaction volume was $l_{\text {gas }}=4.5 \mathrm{~mm}$ [16], resulting in a total number of atoms in the interaction volume $V_{\text {gas }}=S(0) l_{\text {gas }}$ of $\approx 6 \times 10^{10}$. In the case of the cluster experiment for data presented in Fig. 2, the number of atoms in the interaction volume $V=$ $S\left(z_{\mathrm{IP}}=4 \mathrm{~mm}\right) l$ is about $\approx 3.5 \times 10^{11}$ [according to concentration given by Eq. (A1)], i.e., six times higher than in the gas experiment. If one compares the number of atoms in the volume of a cross section corresponding to the Fresnel number equal to 1 (which is a relevant measure for the phenomenon of superfluorescence $[15,47])$, the number of Xe atoms in the corresponding volume is about two times higher for the cluster target. The optical density and amount of atoms in the interaction volume are hence not equal but comparable in both experiments.

\section{APPENDIX B: DERIVATION OF EQ. (7)}

Let us assume for simplicity the decoherence rate to be constant. Under the assumption of $\gamma_{d} \gg r_{e}, \gamma_{e}, \gamma_{g}$ the 
spontaneous emission part of Eq. (4) simplifies to

$$
\begin{aligned}
&{\frac{\partial G\left(z, \tau_{1}, \tau_{2}\right)}{\partial z}=}_{\mathrm{sp}} g_{0}\left\{e^{-\frac{\gamma_{d}}{2}\left(\tau_{1}+\tau_{2}\right)} \rho_{\mathrm{ee}}(z, 0)\right. \\
&\left.+\int_{0}^{\min \tau_{1}, \tau_{2}} d \tau^{\prime} e^{-\frac{\gamma_{d}}{2}\left(\tau_{1}+\tau_{2}-2 \tau^{\prime}\right)} \gamma_{d} \rho_{\mathrm{ee}}\left(z, \tau^{\prime}\right)\right\} .
\end{aligned}
$$

We are interested in times much larger than $1 / \gamma_{d}$, hence the first term in (B1) is exponentially damped. In the second term, one can take into account that $\rho_{\mathrm{ee}}$ does not change essentially on the timescale $1 / \gamma_{d}$ and hence can be taken out of the integral. As a result, one obtains

$$
{\frac{\partial G\left(z, \tau_{1}, \tau_{2}\right)}{\partial z}}_{\mathrm{sp}}=g_{0} e^{-\frac{\gamma_{d}}{2}\left|\tau_{1}-\tau_{2}\right|} \rho_{\mathrm{ee}}\left(z, \min \left(\tau_{1}, \tau_{2}\right)\right) .
$$

Within the same assumption about timescales, one can rewrite the term in the stimulated emission term in Eq. (4) as

$$
\begin{aligned}
& \int_{0}^{\tau_{1}} d \tau_{1}^{\prime} e^{-\frac{\gamma_{d}}{2}\left(\tau_{1}-\tau_{1}^{\prime}\right)} \rho_{\text {inv }}\left(z, \tau_{1}^{\prime}\right) G\left(z, \tau_{1}^{\prime}, \tau_{2}\right) \\
& \approx \rho_{\text {inv }}\left(z, \tau_{1}\right) \int_{0}^{\tau_{1}} d \tau_{1}^{\prime} e^{-\frac{\gamma_{d}}{2}\left(\left[\tau_{1}-\tau_{2}\right]-\left[\tau_{1}^{\prime}-\tau_{2}\right]\right)} \\
& \quad \times G_{s}\left(z,\left(\tau_{1}^{\prime}+\tau_{2}\right) / 2, \tau_{1}^{\prime}-\tau_{2}\right) \\
& \approx \rho_{\text {inv }}(z, \tau) \int_{0}^{\infty} d \Delta \tau^{\prime} e^{-\frac{\gamma_{d}}{2}\left(\Delta \tau-\Delta \tau^{\prime}\right)} G_{s}\left(z, \tau, \Delta \tau^{\prime}\right) .
\end{aligned}
$$

Here, it was assumed that $\Delta \tau \ll \tau_{1} \approx \tau_{2} \approx \tau$. Combining (B3) with a similar term for $\tau_{2}$ and taking into account that convolution in the time domain corresponds to a product in the frequency domain, one arrives at Eq. (7).

\section{APPENDIX C: ESTIMATE OF THE INFLUENCE OF THE EMITTED FIELD ON THE OCCUPATION OF ATOMIC LEVELS}

Let us estimate the change of population inversion when the atom $a$ is exposed to a field with correlation function $G\left(z_{a}, t, \omega\right)$. Following the notations of Ref. [15], the evolution of population inversion is expressed as

$$
\frac{d\left\langle\hat{\sigma}_{z}^{(a)}(\tau)\right\rangle}{d \tau}=\frac{i e}{m \hbar c} p^{*}\left\langle\hat{A}_{-}^{(a)}(\tau) \hat{\sigma}_{-}^{(a)}(\tau)\right\rangle+\text { c.c. }
$$

Here we have omitted spontaneous decay and other pump/decay channels. $\hat{\sigma}_{z}^{(a)}$ is the operator of population inversion, $\hat{\sigma}_{-}^{(a)}$ is the operator for transition from excited to ground state (atomic coherence operator) for atom $a$, and $\vec{p}$ is the dipole moment matrix element. The evolution of atomic coherence can be expressed in quadrature via the field vector potential as [see Ref. [15], Eqs. (C3) and Eq. (D12)]

$$
\hat{\sigma}_{-}^{(a)}(\tau)=\int_{0}^{\tau} d \tau^{\prime} e^{-\frac{\gamma_{d}}{2}\left(\tau-\tau^{\prime}\right)} \frac{2 i e p}{\hbar m c} \hat{\sigma}_{z}^{(a)}\left(\tau^{\prime}\right) \hat{A}_{+}^{(a)}\left(\tau^{\prime}\right)+\hat{\xi}_{-}^{(a)}(\tau),
$$

where $\xi$ is a noise operator that will be averaged and will have no contribution in further considerations. Substituting
Eq. (C2) into Eq. (C1), using Eq. (3), one obtains

$$
\begin{aligned}
\frac{d\left\langle\hat{\sigma}_{z}^{(a)}(\tau)\right\rangle}{d \tau}= & \frac{-2 e^{2}|p|^{2} \Delta o}{m^{2} \hbar c \epsilon_{0} \omega} \int_{0}^{\tau} d \tau^{\prime} e^{-\frac{\gamma_{d}}{2}\left(\tau-\tau^{\prime}\right)}\left\langle\hat{\sigma}_{z}^{(a)}\left(\tau^{\prime}\right)\right\rangle \\
& \times G\left(z_{a}, \tau, \tau^{\prime}\right) \\
\approx & -\frac{3 \pi c^{2}}{\omega^{2}}\left\langle\hat{\sigma}_{z}^{(a)}(\tau)\right\rangle \Gamma_{\mathrm{sp}} \int_{-\infty}^{\infty} d \omega G\left(z_{a}, \tau, \omega\right) \\
& \times \Delta o \frac{\gamma_{d}(z, \tau)}{\left[\gamma_{d}(z, \tau) / 2\right]^{2}+\omega^{2}} .
\end{aligned}
$$

Here we have assumed that $\hat{\sigma}_{z}$ can be factorized out of field variables. In the second line we have used the definition of the spontaneous emission rate and have used similar steps to those in Eq. (B3). If we assume that due to gain narrowing the spectral width of $G\left(z_{a}, \tau, \omega\right)$ is smaller than $\gamma_{d}$, then the integration over frequency will be directly connected with the total intensity. The integral over time is in turn connected with the total emitted photon number $N_{\mathrm{ph}}$, hence one can obtain from Eq. (C3) the following expression for the relative change of population inversion:

$$
\frac{\Delta\left\langle\hat{\sigma}_{z}^{(a)}\right\rangle}{\left\langle\hat{\sigma}_{z}^{(a)}\right\rangle} \approx-\frac{3}{\pi} N_{\mathrm{ph}} \frac{\lambda^{2}}{S_{b}} \frac{\Gamma_{\mathrm{sp}}}{\gamma_{d}} .
$$

For the typical parameters of the studied system, this parameter is about $10^{-6}$, hence the influence of the field on the evolution of the atomic level population can be safely neglected.

\section{APPENDIX D: ANALYTICAL EXPRESSION FOR THE FIELD CORRELATION FUNCTION IN THE CASE OF CONSTANT ATOMIC LEVEL POPULATION}

Consider a simplified version of Eq. (4) in the case when both $\rho_{\text {ee }}$ and $\rho_{\text {inv }}$ can be assumed constant in space and time:

$$
\begin{aligned}
\frac{\partial G\left(z, \tau_{1}, \tau_{2}\right)}{\partial z}= & -\kappa G\left(z, \tau_{1}, \tau_{2}\right) \\
& +g_{1}\left[\int_{0}^{\tau_{1}} d \tau_{1}^{\prime} e^{-\frac{\gamma_{d}}{2}\left(\tau_{1}-\tau_{1}^{\prime}\right)} G\left(z, \tau_{1}^{\prime}, \tau_{2}\right)\right. \\
& \left.+\int_{0}^{\tau_{2}} d \tau_{2}^{\prime} e^{-\frac{\gamma_{d}}{2}\left(\tau_{2}-\tau_{2}^{\prime}\right)} G\left(z, \tau_{1}, \tau_{2}^{\prime}\right)\right] \\
& +g_{0} e^{-\frac{\gamma_{d}}{2}\left|\tau_{1}-\tau_{2}\right|} .
\end{aligned}
$$

Here we have absorbed $\rho_{\mathrm{ee}}$ and $\rho_{\text {inv }}$ into the definitions of $g_{0}$ and $g_{1}$, respectively. Within the semiclassical treatment of the superfluorescence, the evolution of the field is typically described by Maxwell-Bloch equations [47,76]. In the case of constant populations of excited and ground states, an analytical solution for the field amplitude and atomic polarization can be obtained [77]. Treating the initial values of polarization as quantum-mechanical operators, one can arrive at the following expression for the field correlation function:

$$
\begin{aligned}
G\left(z, \tau_{1}, \tau_{2}\right)= & g_{0} e^{-\frac{\gamma_{d}}{2}\left(\tau_{1}+\tau_{2}\right)} \int_{0}^{z} d z^{\prime} e^{-\kappa\left(z-z^{\prime}\right)} \\
& \times\left[I_{0}\left[2 \sqrt{g_{1} \tau_{1}\left(z-z^{\prime}\right)}\right] I_{0}\left[2 \sqrt{g_{1} \tau_{2}\left(z-z^{\prime}\right)}\right]\right.
\end{aligned}
$$




$$
\begin{aligned}
& +\int_{0}^{\min \tau_{1}, \tau_{2}} d \tau^{\prime} \gamma_{d} e^{\gamma_{d} \tau^{\prime}} I_{0}\left[2 \sqrt{g_{1}\left(\tau_{1}-\tau^{\prime}\right)\left(z-z^{\prime}\right)}\right] I_{0} \\
& \left.\times\left[2 \sqrt{g_{1}\left(\tau_{2}-\tau^{\prime}\right)\left(z-z^{\prime}\right)}\right]\right] .
\end{aligned}
$$

From Eq. (D2) one can directly obtain the expression for intensity $I(z, \tau)=G(z, \tau, \tau)$. For times much larger than the decoherence time, the first term in Eq. (D2) disappears, and the second arrives at a stationary value

$$
I(z)=g_{0} \int_{0}^{z} d z^{\prime} e^{-\kappa\left(z-z^{\prime}\right)} e^{\frac{2 g_{1}\left(z-z^{\prime}\right)}{\gamma_{d}}} I_{0}\left[2 g_{1}\left(z-z^{\prime}\right) / \gamma_{d}\right]
$$

after a time of about $\sim g_{1} z / \gamma_{d}$, as was discussed in Refs. [68,76,78].

\section{APPENDIX E: SIMPLIFIED RATE EQUATIONS IN THE COLLISION-FREE CASE FOR STATES INVOLVED IN POPULATION INVERSION}

Let us restrict ourselves to the population of the ground state of neutral Xe $p_{0}$, the core-excited state $p_{c}\left(\mathrm{Xe}^{+}\right)$, and the Auger pumped $\left(\mathrm{Xe}^{2+}\right)$ upper $p_{e}$ and lower $p_{g}$ states; see the sketch of the levels in Fig. 18. The resulting rate equations are given by Eqs. (1) without terms $\left(\frac{d p_{0, c, e, g}}{d t}\right)_{\text {coll. }}$.

Since the pumping process is much shorter than the subsequent radiation process, we are interested in populations $p_{e}, p_{g}$ after the pump pulse. Under reasonable assumptions, these quantities can be obtained in terms of simple analytical expressions for any pulse shape. Namely, consider a combination of equations following from Eq. (1):

$$
\frac{d p_{0}}{d t}=-\sigma_{P} j(t) p_{0},
$$

$$
\frac{d\left(p_{c}+p_{e, g} / b_{e, g}\right)}{d t}=\sigma_{P} j(t) p_{0}-\sigma_{P} j(t)\left(p_{c}+p_{e, g} / b_{e, g}\right) .
$$

The solution of Eq. (E1) is

$p_{c}(t)+p_{e}(t) / b_{e}=\int_{-\infty}^{t} d t^{\prime} \sigma_{P} j\left(t^{\prime}\right) \exp \left(-\int_{-\infty}^{t} d t^{\prime} \sigma_{P} j\left(t^{\prime}\right)\right)$.

Assuming that $p_{c}$ decays fast due to the Auger decay, one has

$$
p_{e, g}=b_{e, g} N_{P} \frac{\sigma_{P}}{S_{b}} \exp \left(-N_{P} \frac{\sigma_{P}}{S_{b}}\right)
$$

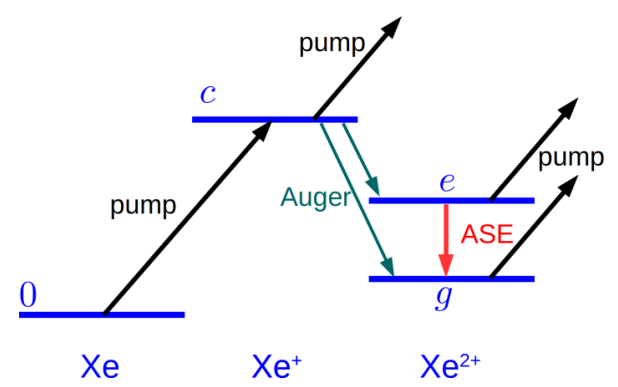

FIG. 18. Sketch of levels involved in simplified rate equations in the collision-free case. where $N_{P}$ is total number of photons in the pump pulse, and $S_{b}$ is the pump beam cross section. As seen from Eq. (E3), the occurrence of the population inversion in this model is conditioned only by the ratio of Auger branching ratios $b_{e}, b_{g}$.

\section{APPENDIX F: INCLUSION OF THE FOUR-LEVEL SCHEME INTO THE CONFIGURATION-AVERAGE COLLISIONAL-RADIATIVE MODEL}

The atomic physics needed for calculating the hydrodynamic evolution of a cluster plasma is necessarily coarsegrained. The reason is that one cannot follow in time and, in each cell, a huge number of fine-structure levels ( $J$-levels). This would involve too large collisional-radiative models to be solved. To describe ASE in Xe clusters, we use a four-level model described in Appendix E (with the proper photoionization and Auger rates) and add, for each of the four equations, an external damping rate and an external source term. These last two quantities correspond to the possible collisional and radiative couplings with the other levels defined in the extended model intended for hydrodynamic calculations.

The set of equations describing the evolution of atomic populations is separated into two systems:

(i) On the one hand, a system for the few detailed levels specifically involved in the ASE scheme and whose populations are strongly affected by the pumping and Auger decay (described in Appendix E). Because of the population inversion arising between these levels, a fine evaluation of their populations is needed.

(ii) On the other hand, a system for all levels whose populations participate in the $\mathrm{x}$-ray absorption, ionization properties, internal energy of the system, and which are necessary for the description of hydrodynamics. These levels (and their collisional and radiative coupling rates; see Ref. [64]) are considered at the configuration average (CA) approximation.

In that sense, system (ii) is independent. The coupling of (i) with (ii) is realized by adding damping rates and source terms due to the couplings with the (other) levels of system (ii) to equations of system (i). The construction of these terms obeys the following rules (see Ref. [64]). Consider the set of levels $i$ belonging to configuration $c$ and the set of levels $j$ belonging to configuration $c^{\prime}$. Then, assuming a collisional mixing between detailed levels belonging to the same configuration, a rate connecting configuration $c$ to a detailed level $j$ of $c^{\prime}$ reads

$$
\mathbb{T}_{c j}=\frac{g_{j}}{g_{c^{\prime}}} \mathbb{T}_{c c^{\prime}},
$$

where $g_{j}$ is the statistical weight of level $j$ while $g_{c^{\prime}}$ is the statistical weight of configuration $c^{\prime}$. In that way, the (global) source terms make use of the configurations-to-configuration rates and of the configuration populations obtained when solving system (ii). From (F1), using the detailed balance principle and neglecting the energy spread (compared with $k T_{e}$ ) between levels of the same configuration, one gets

$$
\mathbb{T}_{j c}=\mathbb{T}_{c^{\prime} c} .
$$

A difficulty arises if the detailed levels of system (i) are strongly affected by the configuration interaction effect (which is the case here) since the CA description does not 
consider this aspect. In that case, one can still use linear combinations of rates using the known configuration mixing coefficients.

\section{APPENDIX G: ABSORPTION OF THE CLUSTER GAS}

The pump field is strongly absorbed in Xe gas/clusters, especially at $92 \mathrm{eV}$ (absorption cross section $\sigma_{P}=25.3 \mathrm{Mb}$ according to Ref. [79]). For a large cluster, only the front part interacts with the field, while the Xe atoms that are deep inside the cluster are not irradiated by the field and hence do not contribute to the absorption. Hence, absorption of the same concentration of atoms assembled in a gas of large clusters would be smaller (due to shadowing by other atoms) than that of single-atom gas. This effect is well known in atmospheric optics [80] and can be rigorously described by Mie scattering theory. According to it, the absorption coefficient of gas of clusters with refractive index $m$ at wavelength $\lambda$ is given by [81]

$$
\kappa=n_{\mathrm{cl}} \pi r_{\mathrm{cl}}^{2} Q(x, m), x=2 \pi r_{\mathrm{cl}} / \lambda,
$$

where

$$
\begin{aligned}
Q(x, m) & =\frac{2}{x^{2}} \sum_{k=1}^{\infty}(2 k+1)\left(\operatorname{Re}\left[a_{k}+b_{k}\right]-\left[\left|a_{k}\right|^{2}+\left|b_{k}\right|^{2}\right]\right), \\
a_{k} & =\frac{\psi_{k}(x) \psi_{k}^{\prime}(m x)-m \psi_{k}(m x) \psi_{k}^{\prime}(x)}{\zeta_{k}(x) \psi_{k}^{\prime}(m x)-m \psi_{k}(m x) \zeta_{k}^{\prime}(x)} \\
b_{k} & =\frac{m \psi_{k}(x) \psi_{k}^{\prime}(m x)-\psi_{k}(m x) \psi_{k}^{\prime}(x)}{m \zeta_{k}(x) \psi_{k}^{\prime}(m x)-\psi_{k}(m x) \zeta_{k}^{\prime}(x)} \\
\psi_{k}(x) & =x j_{k}(x), \quad \zeta_{k}(x)=x h_{k}^{(2)}(x) .
\end{aligned}
$$

Here, $j_{k}(x)$ is the spherical Bessel function and $h_{k}^{(2)}(x)$ is the spherical Hankel function of the second kind, $n_{\mathrm{cl}}=n / N_{\mathrm{cl}}$ is the concentration of clusters. The absorption coefficient calculated according to Eq. (G1) was used for the calculation of amplified spontaneous emission, and atomic absorption cross sections were taken according to Ref. [79].

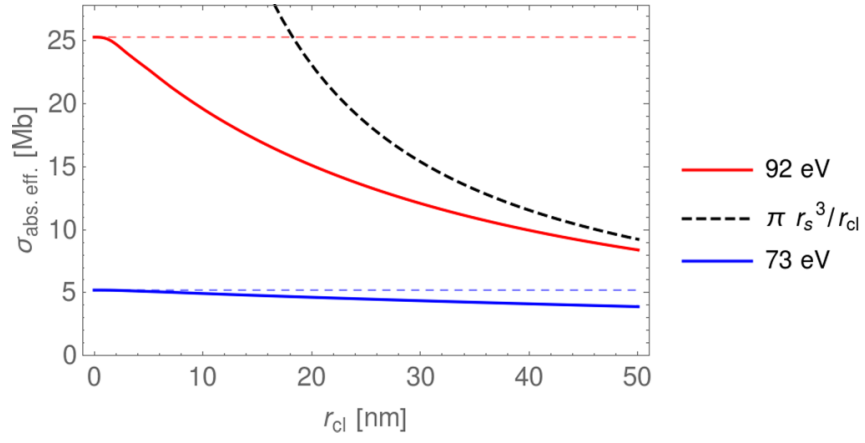

FIG. 19. Dependence of the effective absorption cross section $\sigma_{\text {abs.eff. }}$ on cluster radius $r_{\mathrm{cl}}$. The red solid line corresponds to radiation with $92 \mathrm{eV}$ photon energy, the red dashed line corresponds to the atomic absorption cross section for $92 \mathrm{eV}$, and the blue solid and blue dashed lines correspond to the $73 \mathrm{eV}$ case, respectively. The black dashed line shows the effective absorption cross section for the case of large clusters.

The change of absorption coefficient can be represented as a decrease of the effective absorption cross section of atoms in a mean cluster environment:

$$
\begin{aligned}
\sigma_{\text {abs eff }} & =\pi \frac{r_{s}^{3}}{r_{\mathrm{cl}}} Q\left(2 \pi r_{\mathrm{cl}} / \lambda, m\right) \\
m & =\sqrt{1+m^{\prime \prime 2}}-i m^{\prime \prime}, m^{\prime \prime}=\frac{3}{16 \pi^{2}} \frac{\sigma_{\mathrm{abs}} \lambda}{r_{s}^{3}},
\end{aligned}
$$

where $m$ is the refractive index of a cluster that accounts for absorption by atoms with absorption cross section $\sigma_{\mathrm{abs}}$.

The dependence of the effective absorption cross section on the cluster radius is presented in Fig. 19. It shows that for clusters larger than several $\mathrm{nm}$, the shadowing effect is quite strong for $92 \mathrm{eV}$ pump energy, while for $73 \mathrm{eV}$ pump energy it is not pronounced until about $20 \mathrm{~nm}$ cluster radius. For very large clusters, the absorption cross section of a cluster becomes equal to its geometrical cross section, which corresponds to the atomic effective absorption cross section $\pi r_{s}^{3} / r_{\mathrm{cl}}$. Figure 19 shows that for $92 \mathrm{eV}$ pump energy, the clusters with a radius larger than $50 \mathrm{~nm}$ are already close to this limit.
[1] J. Rossbach, J. R. Schneider, and W. Wurth, 10 years of pioneering X-ray science at the free-electron laser FLASH at DESY, Phys. Rep. 808, 1 (2019).

[2] J. Feldhaus, M. Krikunova, M. Meyer, Th. Möller, R. Moshammer, A. Rudenko, Th. Tschentscher, and J. Ullrich, AMO science at the FLASH and European XFEL free-electron laser facilities, J. Phys. B 46, 164002 (2013).

[3] C. Bostedt, S. Boutet, D. M. Fritz, Z. Huang, H. J. Lee, H. T. Lemke, A. Robert, W. F. Schlotter, J. J. Turner, and G. J. Williams, Linac coherent light source: The first five years, Rev. Mod. Phys. 88, 015007 (2016).

[4] N. Rohringer and R. Santra, X-ray nonlinear optical processes using a self-amplified spontaneous emission free-electron laser, Phys. Rev. A 76, 033416 (2007).
[5] L. Young, E. P. Kanter, B. Krässig, Y. Li, A. M. March, S. T. Pratt, R. Santra, S. H. Southworth, N. Rohringer, L. F. DiMauro, G. Doumy, C. A. Roedig, N. Berrah, L. Fang, M. Hoener, P. H. Bucksbaum, J. P. Cryan, S. Ghimire, J. M. Glownia, D. A. Reis, J. D. Bozek, C. Bostedt, and M. Messerschmidt, Femtosecond electronic response of atoms to ultra-intense X-rays, Nature (London) 466, 56 (2010).

[6] A. A. Sorokin, S. V. Bobashev, T. Feigl, K. Tiedtke, H. Wabnitz, and M. Richter, Photoelectric Effect at Ultrahigh Intensities, Phys. Rev. Lett. 99, 213002 (2007).

[7] M. Arbeiter and T. Fennel, Rare-gas clusters in intense VUV, XUV and soft X-ray pulses: signatures of the transition from nanoplasma-driven cluster expansion to Coulomb explosion in ion and electron spectra, New J. Phys. 13, 053022 (2011). 
[8] R. Santra and L. Young, Interaction of intense x-ray beams with atoms, in Synchrotron Light Sources and Free-Electron Lasers, edited by E. J. Jaeschke, S. Khan, J. R. Schneider, and J. B. Hastings (Springer International, Cham, 2016), pp. 1233-1260.

[9] C. Bostedt, T. Gorkhover, D. Rupp, and T. Möller, Clusters and nanocrystals, in Synchrotron Light Sources and Free-Electron Lasers, edited by E. J. Jaeschke, S. Khan, J. R. Schneider, and J. B. Hastings (Springer International, Cham, 2016), pp. 1323-1364.

[10] N. Rohringer, D. Ryan, R. A. London, M. Purvis, F. Albert, J. Dunn, J. D. Bozek, C. Bostedt, A. Graf, R. Hill, S. P. HauRiege, and J. J. Rocca, Atomic inner-shell X-ray laser at 1.46 nanometres pumped by an X-ray free-electron laser, Nature (London) 481, 488 (2012).

[11] C. Weninger, M. Purvis, D. Ryan, R. A. London, J. D. Bozek, C. Bostedt, A. Graf, G. Brown, J. J. Rocca, and N. Rohringer, Stimulated Electronic X-Ray Raman Scattering, Phys. Rev. Lett. 111, 233902 (2013).

[12] T. Kroll, C. Weninger, R. Alonso-Mori, D. Sokaras, D. Zhu, L. Mercadier, V. P. Majety, A. Marinelli, A. Lutman, M. W. Guetg, F.-J. Decker, S. Boutet, A. Aquila, J. Koglin, J. Koralek, D. P. DePonte, J. Kern, F. D. Fuller, E. Pastor, T. Fransson, Y. Zhang, J. Yano, V. K. Yachandra, N. Rohringer, and U. Bergmann, Stimulated X-Ray Emission Spectroscopy in Transition Metal Complexes, Phys. Rev. Lett. 120, 133203 (2018).

[13] H. Yoneda, Y. Inubushi, K. Nagamine, Y. Michine, H. Ohashi, H. Yumoto, K. Yamauchi, H. Mimura, H. Kitamura, T. Katayama, T. Ishikawa, and M. Yabashi, Atomic inner-shell laser at 1.5-ångström wavelength pumped by an X-ray freeelectron laser, Nature (London) 524, 446 (2015).

[14] C. Weninger and N. Rohringer, Transient-gain photoionization X-ray laser, Phys. Rev. A 90, 063828 (2014).

[15] A. Benediktovitch, V. P. Majety, and N. Rohringer, Quantum theory of superfluorescence based on two-point correlation functions, Phys. Rev. A 99, 013839 (2019).

[16] L. Mercadier, A. Benediktovitch, C. Weninger, M. A. Blessenohl, S. Bernitt, H. Bekker, S. Dobrodey, A. SanchezGonzalez, B. Erk, C. Bomme, R. Boll, Z. Yin, V. P. Majety, R. Steinbrügge, M. A. Khalal, F. Penent, J. Palaudoux, P. Lablanquie, A. Rudenko, D. Rolles, J. R. Crespo López-Urrutia, and N. Rohringer, Evidence of Extreme Ultraviolet Superfluorescence in Xenon, Phys. Rev. Lett. 123, 023201 (2019).

[17] B. Nagler, U. Zastrau, R. R. Fäustlin, S. M. Vinko, T. Whitcher, A. J. Nelson, R. Sobierajski, J. Krzywinski, J. Chalupsky, E. Abreu, S. Bajt, T. Bornath, T. Burian, H. Chapman, J. Cihelka, T. Döppner, S. Düsterer, T. Dzelzainis, M. Fajardo, E. Förster, C. Fortmann, E. Galtier, S. H. Glenzer, S. Göde, G. Gregori, V. Hajkova, P. Heimann, L. Juha, M. Jurek, F. Y. Khattak, A. R. Khorsand, D. Klinger, M. Kozlova, T. Laarmann, H. J. Lee, R. W. Lee, K.-H. Meiwes-Broer, P. Mercere, W. J. Murphy, A. Przystawik, R. Redmer, H. Reinholz, D. Riley, G. Röpke, F. Rosmej, K. Saksl, R. Schott, R. Thiele, J. Tiggesbäumker, S. Toleikis, T. Tschentscher, I. Uschmann, H. J. Vollmer, and J. S. Wark, Turning solid aluminium transparent by intense soft X-ray photoionization, Nat. Phys. 5, 693 (2009).

[18] S. Toleikis, R. R. Fäustlin, L. Cao, T. Döppner, S. Düsterer, E. Förster, C. Fortmann, S. H. Glenzer, S. Göde, G. Gregori, R. Irsig, T. Laarmann, H. J. Lee, B. Li, J. Mithen, K.-H. MeiwesBroer, A. Przystawik, P. Radcliffe, R. Redmer, F. Tavella, R.
Thiele, J. Tiggesbärumker, N. X. Truong, I. Uschmann, U. Zastrau, and Th. Tschentscher, Soft X-ray scattering using FEL radiation for probing near-solid density plasmas at few electron volt temperatures, High Energy Density Phys. 6, 15 (2010).

[19] F. Graziani, M. P. Desjarlais, R. Redmer, and S. B. Trickey, Frontiers and Challenges in Warm Dense Matter (Springer Science \& Business, Cham, 2014), Vol. 96.

[20] S. Suckewer and P. Jaeglé, X-ray laser: past, present, and future, Laser Phys. Lett. 6, 411 (2009).

[21] B. E. Lemoff, G. Y. Yin, C. L. Gordon III, C. P. J. Barty, and S. E. Harris, Demonstration of a 10-Hz Femtosecond-PulseDriven XUV Laser at $41.8 \mathrm{~nm}$ in Xe IX, Phys. Rev. Lett. 74, 1574 (1995).

[22] S. Sebban, R. Haroutunian, Ph. Balcou, G. Grillon, A. Rousse, S. Kazamias, T. Marin, J. P. Rousseau, L. Notebaert, M. Pittman, J. P. Chambaret, A. Antonetti, D. Hulin, D. Ros, A. Klisnick, A. Carillon, P. Jaeglé, G. Jamelot, and J. F. Wyart, Saturated Amplification of a Collisionally Pumped OpticalField-Ionization Soft X-Ray Laser at $41.8 \mathrm{~nm}$, Phys. Rev. Lett. 86, 3004 (2001).

[23] S. Sebban, T. Mocek, D. Ros, L. Upcraft, Ph. Balcou, R. Haroutunian, G. Grillon, B. Rus, A. Klisnick, A. Carillon, G. Jamelot, C. Valentin, A. Rousse, J. P. Rousseau, L. Notebaert, M. Pittman, and D. Hulin, Demonstration of a NiLike Kr Optical-Field-Ionization Collisional Soft X-Ray Laser at $32.8 \mathrm{~nm}$, Phys. Rev. Lett. 89, 253901 (2002).

[24] M. A. Purvis, V. N. Shlyaptsev, R. Hollinger, C. Bargsten, A. Pukhov, A. Prieto, Y. Wang, B. M. Luther, L. Yin, S. Wang, and J. J. Rocca, Relativistic plasma nanophotonics for ultrahigh energy density physics, Nat. Photon. 7, 796 (2013).

[25] V. Kaymak, A. Pukhov, V. N. Shlyaptsev, and J. J. Rocca, Nanoscale Ultradense Z-pinch Formation from Laser-Irradiated Nanowire Arrays, Phys. Rev. Lett. 117, 035004 (2016).

[26] Y. Dong, J. Yang, T. Song, W. Shang, L. Zhang, C. Huang, T. Zhu, W. Zhang, Z. Li, X. Zhan, H. Du, F. Wang, S. Liu, S. Jiang, and Y. Ding, Efficient soft X-ray sources from laser-irradiated gold foam targets with well-controlled impurities, Nucl. Fusion 58, 016038 (2017).

[27] P. P. Rajeev, P. Taneja, P. Ayyub, A. S. Sandhu, and G. Ravindra Kumar, Metal Nanoplasmas as Bright Sources of Hard X-Ray Pulses, Phys. Rev. Lett. 90, 115002 (2003).

[28] H. A. Sumeruk, S. Kneip, D. R. Symes, I. V. Churina, A. V. Belolipetski, T. D. Donnelly, and T. Ditmire, Control of Strong-Laser-Field Coupling to Electrons in Solid Targets with Wavelength-Scale Spheres, Phys. Rev. Lett. 98, 045001 (2007).

[29] G. Kulcsár, D. Al Mawlawi, F. W. Budnik, P. R. Herman, M. Moskovits, L. Zhao, and R. S. Marjoribanks, Intense Picosecond X-Ray Pulses from Laser Plasmas by use of Nanostructured "Velvet" Targets, Phys. Rev. Lett. 84, 5149 (2000).

[30] T. Ditmire, R. A. Smith, R. S. Marjoribanks, G. Kulcs, and M. H. R. Hutchinson, X-ray yields from Xe clusters heated by short pulse high intensity lasers, Appl. Phys. Lett. 71, 166 (1997).

[31] L. M. Chen, F. Liu, W. M. Wang, M. Kando, J. Y. Mao, L. Zhang, J. L. Ma, Y. T. Li, S. V. Bulanov, T. Tajima, Y. Kato, Z. M. Sheng, Z. Y. Wei, and J. Zhang, Intense High-Contrast Femtosecond $K$-Shell X-Ray Source from Laser-Driven $\mathrm{Ar}$ Clusters, Phys. Rev. Lett. 104, 215004 (2010).

[32] C. Stenz, V. Bagnoud, F. Blasco, J. R. Roche, F. Salin, A. Ya. Faenov, A. I. Magunov, T. A. Pikuz, and I. Yu. Skobelev, X-ray 
emission spectra of the plasma produced by an ultrashort laser pulse in cluster targets, Quantum Electron. 30, 721 (2000).

[33] L. M. Chen, W. C. Yan, D. Z. Li, Z. D. Hu, L. Zhang, W. M. Wang, N. Hafz, J. Y. Mao, K. Huang, Y. Ma, J. R. Zhao, J. L. Ma, Y. T. Li, X. Lu, Z. M. Sheng, Z. Y. Wei, J. Gao, and J. Zhang, Bright betatron X-ray radiation from a laser-drivenclustering gas target, Sci. Rep. 3, 1912 (2013).

[34] V. Y. Banine, K. N. Koshelev, and G. H. P. M. Swinkels, Physical processes in EUV sources for microlithography, J. Phys. D 44, 253001 (2011).

[35] R. Schupp, F. Torretti, R. A. Meijer, M. Bayraktar, J. Scheers, D. Kurilovich, A. Bayerle, K. S. E. Eikema, S. Witte, W. Ubachs, R. Hoekstra, and O. O. Versolato, Efficient Generation of Extreme Ultraviolet Light from Nd:YAG-Driven Microdroplet-Tin Plasma, Phys. Rev. Appl. 12, 014010 (2019).

[36] B. Erk, J. P. Müller, C. Bomme, R. Boll, G. Brenner, H. N. Chapman, J. Correa, S. Düsterer, S. Dziarzhytski, S. Eisebitt, H. Graafsma, S. Grunewald, L. Gumprecht, R. Hartmann, G. Hauser, B. Keitel, C. v. K. Schmising, M. Kuhlmann, B. Manschwetus, L. Mercadier, E. Müller, C. Passow, E. Plönjes, D. Ramm, D. Rompotis, A. Rudenko, D. Rupp, M. Sauppe, F. Siewert, D. Schlosser, L. Strüder, A. Swiderski, S. Techert, K. Tiedtke, T. Tilp, R. Treusch, I. Schlichting, J. Ullrich, R. Moshammer, T. Möller, and D. Rolles, CAMP@FLASH: An end-station for imaging, electron- and ion-spectroscopy, and pump-probe experiments at the FLASH free-electron laser, J. Synch. Radiat. 25, 1529 (2018).

[37] D. Rupp, Ionization and Plasma Dynamics of Single Large Xenon Clusters in Superintense XUV Pulses (Springer, Cham, 2016).

[38] L. Schroedter, M. Müller, A. Kickermann, A. Przystawik, S. Toleikis, M. Adolph, L. Flückiger, T. Gorkhover, L. Nösel, M. Krikunova, T. Oelze, Y. Ovcharenko, D. Rupp, M. Sauppe, D. Wolter, S. Schorb, C. Bostedt, T. Möller, and T. Laarmann, Hidden Charge States in Soft-X-Ray Laser-Produced Nanoplasmas Revealed by Fluorescence Spectroscopy, Phys. Rev. Lett. 112, 183401 (2014).

[39] J. Jauhiainen, H. Aksela, S. Aksela, A. Kivimaki, O. P. Sairanen, E. Nommiste, and J. Vegh, Correlation satellites in the $\mathrm{Xe}_{4,5}$-OO and $\mathrm{Kr} \mathrm{M}_{4,5}$-NN Auger spectra, J. Phys. B 28, 3831 (1995).

[40] J. Wörmer, V. Guzielski, J. Stapelfeldt, and T. Möller, Fluorescence excitation spectroscopy of xenon clusters in the VUV, Chem. Phys. Lett. 159, 321 (1989).

[41] A. Przystawik, L. Schroedter, M. Müller, M. Adolph, C. Bostedt, L. Flückiger, T. Gorkhover, A. Kickermann, M. Krikunova, L. Nösel, T. Oelze, Y. Ovcharenko, D. Rupp, M. Sauppe, S. Schorb, S. Usenko, T. Möller, and T. Laarmann, Ionization dynamics of Xe nanoplasma formation studied with XUV fluorescence spectroscopy, J. Phys. B 48, 184002 (2015).

[42] M. Müller, L. Schroedter, T. Oelze, L. Nösel, A. Przystawik, A. Kickermann, M. Adolph, T. Gorkhover, L. Flückiger, M. Krikunova, M. Sauppe, Y. Ovcharenko, S. Schorb, C. Bostedt, D. Rupp, T. Laarmann, and T. Möller, Ionization dynamics of XUV excited clusters: the role of inelastic electron collisions, J. Phys. B 48, 174002 (2015).

[43] E. Ackad, N. Bigaouette, and L. Ramunno, Augmented collisional ionization via excited states in XUV cluster interactions, J. Phys. B 44, 165102 (2011).
[44] O. F. Hagena, Nucleation and growth of clusters in expanding nozzle flows, Surf. Sci. 106, 101 (1981).

[45] J. A. Koch, B. J. MacGowan, L. B. Da Silva, D. L. Matthews, J. H. Underwood, P. J. Batson, and S. Mrowka, Observation of Gain-Narrowing and Saturation Behavior in Se X-Ray Laser Line Profiles, Phys. Rev. Lett. 68, 3291 (1992).

[46] R. H. Dicke, Coherence in spontaneous radiation processes, Phys. Rev. 93, 99 (1954).

[47] M. Gross and S. Haroche, Superradiance: An essay on the theory of collective spontaneous emission, Phys. Rep. 93, 301 (1982).

[48] R. T. Sutherland and F. Robicheaux, Superradiance in inverted multilevel atomic clouds, Phys. Rev. A 95, 033839 (2017).

[49] S. Prasad and Roy J. Glauber, Initiation of superfluorescence in a large sphere, Phys. Rev. A 31, 1583 (1985).

[50] I. I. Sobel'Man, L. A. Vainshtein, and E. A. Yukov, Excitation of Atoms and Broadening of Spectral Lines (Springer-Verlag, Berlin, 1995), Vol. 15.

[51] A. Yariv and R. C. C. Leite, Super radiant narrowing in fluorescence radiation of inverted populations, J. Appl. Phys. 34, 3410 (1963).

[52] G. J. Linford, E. R. Peressini, W. R. Sooy, and M. L. Spaeth, Very long lasers, Appl. Opt. 13, 379 (1974).

[53] O. Svelto, S. Taccheo, and C. Svelto, Analysis of amplified spontaneous emission: some corrections to the Linford formula, Opt. Commun. 149, 277 (1998).

[54] J. J. Rocca, Table-top soft X-ray lasers, Rev. Sci. Instrum. 70, 3799 (1999).

[55] E. Oliva, M. Fajardo, L. Li, M. Pittman, T. T. T. Le, J. Gautier, G. Lambert, P. Velarde, D. Ros, S. Sebban, and Ph. Zeitoun, A proposal for multi-tens of GW fully coherent femtosecond soft X-ray lasers, Nat. Photon. 6, 764 (2012).

[56] B. Ziaja, H. Wabnitz, E. Weckert, and T. Möller, Femtosecond non-equilibrium dynamics of clusters irradiated with short intense VUV pulses, New J. Phys. 10, 043003 (2008).

[57] U. Saalmann, Ch. Siedschlag, and J. M. Rost, Mechanisms of cluster ionization in strong laser pulses, J. Phys. B 39, R39 (2006).

[58] Th. Fennel, K.-H. Meiwes-Broer, J. Tiggesbäumker, P.-G. Reinhard, P. M. Dinh, and E. Suraud, Laser-driven nonlinear cluster dynamics, Rev. Mod. Phys. 82, 1793 (2010).

[59] M. Arbeiter, C. Peltz, and T. Fennel, Electron-relocalization dynamics in xenon clusters in intense soft-X-ray fields, Phys. Rev. A 89, 043428 (2014).

[60] Z. Jurek, S.-K. Son, B. Ziaja, and R. Santra, $X M D Y N$ and XATOM: Versatile simulation tools for quantitative modeling of $\mathrm{X}$-ray free-electron laser induced dynamics of matter, J. Appl. Crystallogr. 49, 1048 (2016).

[61] P. J. Ho and C. Knight, Large-scale atomistic calculations of clusters in intense X-ray pulses, J. Phys. B 50, 104003 (2017).

[62] O. Peyrusse, Coupling of detailed configuration kinetics and hydrodynamics in materials submitted to X-ray free-electronlaser irradiation, Phys. Rev. E 86, 036403 (2012).

[63] O. Peyrusse, J.-M. André, P. Jonnard, and J. Gaudin, Modeling of the interaction of an X-ray free-electron laser with large finite samples, Phys. Rev. E 96, 043205 (2017).

[64] O. Peyrusse, Atomic configuration averages and non-local thermodynamical equilibrium plasma spectroscopy calculations, J. Phys. B 32, 683 (1999). 
[65] P. Andersen, T. Andersen, F. Folkmann, V. K. Ivanov, H. Kjeldsen, and J. B. West, Absolute cross sections for the photoionization of $4 \mathrm{~d}$ electrons in $\mathrm{Xe}^{+}$and $\mathrm{Xe}^{2+}$ ions, J. Phys. B 34, 2009 (2001).

[66] C. Jungreuthmayer, L. Ramunno, J. Zanghellini, and T. Brabec, Intense VUV laser cluster interaction in the strong coupling regime, J. Phys. B 38, 3029 (2005).

[67] H. Griem, Spectral Line Broadening by Plasmas (Academic Press, New York, 1974).

[68] M. F. H. Schuurmans, Superfluorescence and amplified spontaneous emission in an inhomogeneously broadened medium, Opt. Commun. 34, 185 (1980).

[69] B. Talin, A. Calisti, L. Godbert, R. Stamm, R. W. Lee, and L. Klein, Frequency-fluctuation model for line-shape calculations in plasma spectroscopy, Phys. Rev. A 51, 1918 (1995).

[70] O. F. Hagena, Condensation in free jets: Comparison of rare gases and metals, Z. Phys. D 4, 291 (1987).

[71] R. Karnbach, M. Joppien, J. Stapelfeldt, J. Wörmer, and T. Möller, CLULU: An experimental setup for luminescence measurements on van der waals clusters with synchrotron radiation, Rev. Sci. Instrum. 64, 2838 (1993).

[72] O. F. Hagena, Cluster ion sources (invited), Rev. Sci. Instrum. 63, 2374 (1992).

[73] U. Buck and R. Krohne, Cluster size determination from diffractive He atom scattering, J. Chem. Phys. 105, 5408 (1996).
[74] F. Dorchies, F. Blasco, T. Caillaud, J. Stevefelt, C. Stenz, A. S. Boldarev, and V. A. Gasilov, Spatial distribution of cluster size and density in supersonic jets as targets for intense laser pulses, Phys. Rev. A 68, 023201 (2003).

[75] A. S. Boldarev, V. A. Gasilov, A. Ya. Faenov, Y. Fukuda, and K. Yamakawa, Gas-cluster targets for femtosecond laser interaction: Modeling and optimization, Rev. Sci. Instrum. 77, 083112 (2006).

[76] M. G. Benedikt, A. M. Ermolaev, V. A. Malyshev, I. V. Sokolov, and E. D. Trifonov, Super-Radiance: Multiatomic Coherent Emission (Institute of Physics, Bristol, UK, 1996).

[77] J. A. Hermann, An amplifying solution of the Maxwell-Bloch equations with atomic relaxation and field losses, J. Phys. A 13, 3543 (1980).

[78] M. F. H. Schuurmans and D. Polder, Superfluorescence and amplified spontaneous emission: A unified theory, Phys. Lett. A 72, 306 (1979).

[79] J. A. R. Samson and W. C. Stolte, Precision measurements of the total photoionization cross-sections of $\mathrm{He}, \mathrm{Ne}, \mathrm{Ar}$, $\mathrm{Kr}$, and Xe, J. Electron Spectrosc. Relat. Phenom. 123, 265 (2002).

[80] H. Horvath, Atmospheric light absorption-A review, Atm. Envir. Pt. A 27, 293 (1993), First Ibero-American Conference on the Atmospheric Environment, CIAMAA91/ACAE91.

[81] C. F. Bohren and D. R. Huffman, Absorption and Scattering by a Sphere (Wiley, 2007), Chap. 4, pp. 82-129. 